\title{
Simplified Model for Reburning Chemistry
}

\author{
Glarborg, Peter; Hansen, Stine
}

\section{Published in:}

Energy \& Fuels

Link to article, DOI:

10.1021/ef100469h

Publication date:

2010

Document Version

Publisher's PDF, also known as Version of record

Link back to DTU Orbit

Citation (APA):

Glarborg, P., \& Hansen, S. (2010). Simplified Model for Reburning Chemistry. Energy \& Fuels, 24(8), 41854192. https://doi.org/10.1021/ef100469h

\section{General rights}

Copyright and moral rights for the publications made accessible in the public portal are retained by the authors and/or other copyright owners and it is a condition of accessing publications that users recognise and abide by the legal requirements associated with these rights.

- Users may download and print one copy of any publication from the public portal for the purpose of private study or research.

- You may not further distribute the material or use it for any profit-making activity or commercial gain

- You may freely distribute the URL identifying the publication in the public portal

If you believe that this document breaches copyright please contact us providing details, and we will remove access to the work immediately and investigate your claim. 


\title{
Simplified Model for Reburning Chemistry
}

\author{
Stine Hansen and Peter Glarborg* \\ Department of Chemical Engineering, Technical University of Denmark, \\ 2800 Lyngby, Denmark
}

Received April 14, 2010. Revised Manuscript Received July 6, 2010

\begin{abstract}
In solid fuel flames, reburn-type reactions are often important for the concentrations of $\mathrm{NO}_{x}$ in the nearburner region. To be able to model the nitrogen chemistry in these flames, it is necessary to have an adequate model for volatile/NO interactions. Simple models consisting of global steps or based on partial-equilibrium assumptions have limited predictive capabilities. Reburning models based on systematic reduction of a detailed chemical kinetic model offer a high accuracy but rely on input estimates of combustion intermediates, including free radicals. In the present work, an analytically reduced nitrogen scheme is combined with simplified correlations for estimation of $\mathrm{O} / \mathrm{H}$ and hydrocarbon radicals. Correlations are derived for volatile compositions representative of solid fuels ranging from bituminous coal to biomass, for temperatures of $1200-2000 \mathrm{~K}$ and excess air ratios in the range of $0.6 \leq \lambda \leq 2.0$. The combined model is tested against reference calculations with a comprehensive mechanism. The results indicate that the approximations in the simplified hydrocarbon radical scheme are satisfactory. However, when this scheme is combined with the semi-empirical correlations for the $\mathrm{O} / \mathrm{H}$ radicals, the modeling predictions for the radicals become less accurate. Despite these deviations, the combined model provides a satisfactory prediction of NO under reburning conditions over the range of fuels, temperatures, and stoichiometries tested.
\end{abstract}

\section{Introduction}

Computational fluid dynamic (CFD) models are often used to predict pollutant formation, in particular, nitrogen oxides $\left(\mathrm{NO}_{x}\right)$, in combustion systems. Although significant efforts have been aimed at modeling and understanding NO formation and destruction, it remains a challenge to quantitatively predict $\mathrm{NO}$ emissions from practical systems. Because of the complexity of turbulent combustion, simplified schemes are often required to describe the chemistry, ${ }^{1,2}$ to reduce the computational load. Several simplified approaches for modeling nitrogen chemistry in combustion have been reported. Typically, they involve either an empirical fitting of a set of global reaction parameters to experimental data or an analytical reduction of comprehensive models through sensitivity analysis and/or equilibrium considerations.

Simplified schemes developed to predict volatile $\mathrm{N}$ oxidation in combustion include both global model $\mathrm{s}^{3-6}$ and analytically reduced schemes. ${ }^{7,8}$ Under reducing conditions, reburn-type reactions recycling $\mathrm{NO}$ to cyanide species may become important. Here, the nitrogen chemistry is closely coupled to details of the fuel oxidation chemistry, i.e., the

*To whom correspondence should be addressed. Fax: +45-45882258. E-mail:pgl@kt.dtu.dk.

(1) Andersen, J.; Jensen, P. A.; Meyer, K. E.; Hvid, S. L.; Glarborg, P. Energy Fuels 2009, 23, 5773-5782.

(2) Andersen, J.; Jensen, P. A.; Hvid, S. L.; Glarborg, P. Energy Fuels 2009, 23, 5783-5791.

(3) De Soete, G. G. Proc. Combust. Inst. 1975, 15, 1093-1102.

(4) Mitchell, J. W.; Tarbell, J. M. AIChE J. 1982, 28, 302-311.

(5) Chen, W.; Smoot, L. D.; Fletcher, T. H.; Boardman, R. D. Energy Fuels 1996, 10, 1036-1045.

(6) Brink, A.; Kilpinen, P.; Hupa, M. Energy Fuels 2001, 15, 10941099.

(7) Glarborg, P.; Lilleheie, N.; Byggstøyl, S.; Magnussen, B.; Kilpinen, P.; Hupa, M. Proc. Combust. Inst. 1992, 24, 889-898.

(8) Pedersen, L. S.; Glarborg, P.; Dam-Johansen, K. Combust. Sci. Technol. 1998, 131, 193-223. formation and destruction of hydrocarbon radicals, and the prediction of NO constitutes a demanding test of simplified mechanism concepts. Published schemes for reburning include global mechanisms, ${ }^{9}$ sets based on partial equilibrium approaches, ${ }^{10-13}$ and analytically reduced schemes. ${ }^{7,14-16}$

Two different simplified schemes for NO reduction by reburning reactions are employed in the commercial CFD code Fluent. ${ }^{17}$ Both schemes, termed the instantaneous approach and the partial equilibrium approach, respectively, describe the reduction of $\mathrm{NO}$ by the reaction with the $\mathrm{C}_{1}$ radical pool to form $\mathrm{HCN}$ (or $\mathrm{CN}$ ). The difference between the schemes concerns the choice of radicals and the way their concentration is estimated. The instantaneous approach involves the hydrocarbon radicals $\mathrm{CH}_{3}, \mathrm{CH}_{2}$, and $\mathrm{CH}$; their concentrations are drawn from calculations with a detailed combustion mechanism. The partial equilibrium approach is based on the work by Dimitriou et al. ${ }^{11}$ Here, the principal pathways of $\mathrm{NO}$ reduction are assumed to involve the reaction with the radicals $\mathrm{CH}_{2}, \mathrm{CH}$, and $\mathrm{C}$. The concentration of these radicals are found by assuming the following reactions to be in partial equilibrium: $\mathrm{CH}_{4}+\mathrm{H} \rightleftharpoons \mathrm{CH}_{3}+\mathrm{H}_{2}, \mathrm{CH}_{3}+\mathrm{OH} \rightleftharpoons$

(9) Chen, W.; Smoot, L. D.; Hill, S. C.; Fletcher, T. H. Energy Fuels 1996, 10, 1046-1052.

(10) Mereb, B. J.; Wendt, J. O. L. Proc. Combust. Inst. 1990, 23, 12731279

(11) Dimitriou, D. J.; Kandamby, N.; Lockwood, F. C. Fuel 2003, 82, 2107-2114

(12) Su, S.; Xiang, J.; Sun, X.; Zhang, Z.; Zheng, C.; Xu, M. Energy Fuels 2006, 20, 1434-1443.

(13) Su, S.; Xiang, J.; Sun, L.; Zhang, Z.; Sun, X.; Zheng, C. Proc. Combust. Inst. 2007, 31, 2795-2803.

(14) Giral, I.; Alzueta, M. U. Fuel 2002, 81, 2263-2275.

(15) Han, X.; Rückert, F.; Schnell, U.; Hein, K. R. G.; Koger, S.; Bockhorn, H. Combust. Sci. Technol. 2003, 175, 523-544

(16) Lv, Y.; Wang, Z.; Zhou, J.; Cen, K. Energy Fuels 2009, 23, 59205928

(17) Fluent 6.3 Users Guide; Fluent, Inc.: Lebanon, NH, 2005. 


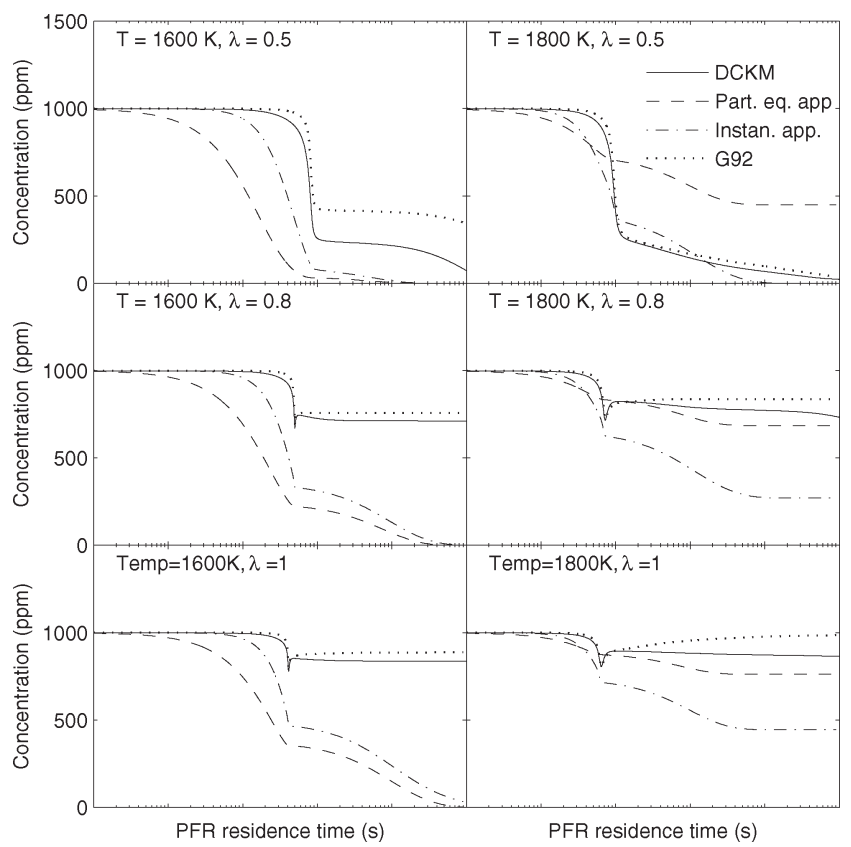

Figure 1. Predictions of NO reburning during combustion of the $\mathrm{CH}_{4}$ in the $\mathrm{N}_{2} / \mathrm{O}_{2}$ mixture with $1 \mathrm{vol} \% \mathrm{O}_{2}$ under isothermal plugflow reactor conditions.

$\mathrm{CH}_{2}+\mathrm{H}_{2} \mathrm{O}, \mathrm{CH}_{2}+\mathrm{H} \rightleftharpoons \mathrm{CH}+\mathrm{H}_{2}$, and $\mathrm{CH}+\mathrm{H} \rightleftharpoons \mathrm{C}+\mathrm{H}_{2}$. The omission of reactions between $\mathrm{CH}_{3}$ and $\mathrm{NO}$ in the partial equilibrium approach would be expected to limit the accuracy, at least at temperatures below $1500 \mathrm{~K}$, where methyl has been shown to be active in reducing NO. ${ }^{18-20}$ It is also noteworthy that neither of the Fluent schemes involves the HCCO radical, which even for combustion of $\mathrm{CH}_{4}$ is predicted to be important for NO removal. ${ }^{18-20}$

Figure 1 compares modeling predictions of NO reburning with the simplified schemes employed in Fluent (instantaneous and partial equilibrium approaches), the analytically reduced scheme by Glarborg et al. ${ }^{7}$ (denoted G92), and the recent detailed chemical kinetic model by Mendiara and Glarborg ${ }^{20,23}$ (denoted DCKM). These calculations, as well as those shown below, were conducted using Chemkin ${ }^{21,22}$ and Matlab, respectively, assuming conditions of an isothermal plug-flow reactor. The calculations show the NO reduction during combustion under isothermal conditions of methane in the $\mathrm{N}_{2} / \mathrm{O}_{2}$ mixture with $1 \mathrm{vol} \% \mathrm{O}_{2}$ at selected excess air ratios $(\lambda)$ and temperatures. To exclude differences in the selected mechanisms caused by anything other than the nitrogen chemistry descriptions, other species concentrations $\left(\mathrm{O}_{2}, \mathrm{H}_{2}\right.$, radicals, etc. $)$ are drawn from the detailed mechanism.

The results of Figure 1 show that the schemes employed by Fluent ${ }^{17}$ have considerable shortcomings. The instantaneous

(18) Glarborg, P.; Alzueta, M. U.; Dam-Johansen, K.; Miller, J. A. Combust. Flame 1998, 115, 1-27.

(19) Skreiberg, Ø.; Kilpinen, P.; Glarborg, P. Combust. Flame 2004, $136,501-518$.

(20) Mendiara, T.; Glarborg, P. Energy Fuels 2009, 23, 3565-3572.

(21) Kee, R. J.; Rupley, F. M.; Miller, J. A. Chemkin II: A Fortran Chemical Kinetics Package for the Analysis of Gas Phase Chemical Kinetics. Sandia Report SAND89-8009B-UC-706; Sandia National Laboratories: Livermore, CA, 1989.

(22) Lutz, A. E.; Kee, R. J.; Miller, J. A. Senkin: A Fortran Program for Predicting Homogeneous Gas Phase Chemical Kinetics With Sensitivity Analysis. Sandia Report SAND87-8248 U UC-401; Sandia National Laboratories: Livermore, CA, 1990.

(23) Mendiara, T.; Glarborg, P. Combust. Flame 2009, 156, 19371949 . approach also predicts a large reduction of $\mathrm{NO}$ at all of the conditions examined, partly because it does not account for recycling of the cyanides to NO. The partial equilibrium approach provides a reasonable description of the NO emission level at the high temperature and $\lambda \geq 0.8$. At more reducing conditions or lower temperatures, the predictions are less accurate. The discrepancies may be attributed partly to the selection of reactions between hydrocarbons and NO and partly to the prediction of hydrocarbon radical concentrations.

Contrary to the Fluent schemes, the reduced mechanism by Glarborg et al. provides a good description of NO reburning at all of the examined conditions, except at very reducing conditions and lower temperatures. A similar level of accuracy would be expected from the more recent analytically reduced reburn schemes. ${ }^{15,16}$ Even though these schemes are superior to the global models in terms of accuracy, their use has thus far been limited because they are computationally more demanding and rely on input estimates of combustion intermediates. In particular, these schemes require estimates of free-radical concentrations, values which in the past have been available only from modeling with either comprehensive mechanisms or analytically reduced fuel oxidation schemes. Global schemes, such as the two-step hydrocarbon oxidation mechanism by Westbrook and Dryer ${ }^{24}$ or the four-step mechanism by Jones and Lindstedt, ${ }^{25}$ offer estimates of the fuel oxidation rate and concentrations of intermediates, such as $\mathrm{CO},{ }^{26}$ but they are unable to predict radical levels.

Recently, ${ }^{27}$ we developed a simple approach for estimating $\mathrm{O} / \mathrm{H}$ radical concentrations and combined it with the analytically reduced $\mathrm{N}$ scheme by Pedersen et al. ${ }^{8}$ to yield a modeling tool for volatile $\mathrm{N}$ oxidation. In the present work, we extend this approach to involve also hydrocarbon radicals, to develop a simplified modeling tool for reburning-type chemistry. Simplified relations are derived for volatile compositions representative of solid fuels ranging from coal to biomass. The combined model, which describes the important gas-phase formation and consumption mechanisms for $\mathrm{NO}^{28,29}$ is tested against reference calculations with a comprehensive mechanism.

\section{Numerical Procedure}

The model developed in the present work consists of three parts: a simple approach for estimating $\mathrm{O} / \mathrm{H}$ radical concentrations (scheme I), a scheme for prediction of hydrocarbon radical concentrations (scheme II), and an analytically reduced mechanism to describe the nitrogen chemistry (scheme III). Scheme I, consisting of semi-empirical correlations for $\mathrm{H}, \mathrm{O}$, and $\mathrm{OH}$, is adopted from previous work. ${ }^{27}$ Scheme II combines an analytically reduced scheme for $\mathrm{C}_{1}$ radicals ${ }^{7}$ with semi-empirical correlations for $\mathrm{C}_{2}$ radicals (present work). Finally, the $\mathrm{NO}_{x}$ model (scheme III) is a combined scheme describing volatile $\mathrm{N}$ oxidation with $\mathrm{HCN}$ and $\mathrm{NH}_{3}$ as intermediates, as well as a reduction of $\mathrm{NO}$ by reburning. It is set up initially by combining the reduced mechanisms of Pedersen et al. ${ }^{8}$ and Glarborg et al. ${ }^{7}$ but modified in the present work. The Pedersen scheme was shown in our

(24) Westbrook, C. K.; Dryer, F. L. Combust. Sci. Technol. 1981, 27, $31-44$.

(25) Jones, W. P.; Lindstedt, R. P. Combust. Flame 1988, 73, 233-249.

(26) Andersen, J.; Rasmussen, C. L.; Giselsson, T.; Glarborg, P. Energy Fuels 2009, 23, 1379-1389.

(27) Hansen, S.; Glarborg, P. Energy Fuels 2010, 24, 2883-2890.

(28) Glarborg, P.; Jensen, A. D.; Johnsson, J. E. Prog. Energy Combust. Sci. 2003, 29, 89-113.

(29) Miller, J. A.; Bowman, C. T. Prog. Energy Combust. Sci. 1989, 15, 287-338. 
Table 1. Estimated Volatile Compositions of the Selected Fuels ${ }^{27}$

\begin{tabular}{crrrr}
\hline & \multicolumn{4}{c}{ composition $(\text { vol \%) })^{a}$} \\
\cline { 2 - 5 } fuel number & \multicolumn{1}{c}{1} & \multicolumn{1}{c}{2} & \multicolumn{1}{c}{3} & \multicolumn{1}{c}{4} \\
\hline $\mathrm{H}_{2} \mathrm{O}$ & 16.5 & 12.5 & 5.0 & 1.7 \\
$\mathrm{CO}$ & 12.3 & 8.5 & 6.9 & 38.2 \\
$\mathrm{CO}_{2}$ & 5.0 & 3.2 & 1.2 & 9.1 \\
$\mathrm{H}_{2}$ & 46.7 & 39.1 & 37.5 & 41.4 \\
$\mathrm{CH}_{4}$ & 3.4 & 4.6 & 6.9 & 9.6 \\
$\mathrm{C}_{2} \mathrm{H}_{4}$ & 1.1 & 1.6 & 2.9 & \\
soot $^{c}$ & 14.9 & 30.5 & 39.7 & 0
\end{tabular}

${ }^{a}$ When the volatile composition is entered into the simulations, the remaining components are normalized to yield $100 \%{ }^{b}$ Fuel types: (1) Montana lignite, ${ }^{30}$ (2) Dietz sub-bituminous coal, ${ }^{31}$ (3) Pittsburgh number 8 bituminous coal, ${ }^{32}$ and (4) biomass (poplar wood). ${ }^{33}{ }^{c}$ Not included in the modeling.

recent work $^{27}$ to provide a good description of the oxidation chemistry of $\mathrm{HCN}$ and $\mathrm{NH}_{3}$, but it does not involve hydrocarbon/ nitrogen interactions. The scheme of Glarborg et al. provides a satisfactory description of $\mathrm{C}_{1} / \mathrm{NO}$ interactions (Figure 1) but excludes reactions of $\mathrm{NH}_{3}$.

Similar to the work by Han et al., ${ }^{15}$ the model is developed for use in solid fuel combustion. The radical schemes are applicable to fuels belonging to four different ranks, ranging from bituminous coal to biomass. The assumed volatile compositions of these fuels are listed in Table 1. For each of the fuels, a set of semiempirical equations describing the formation and consumption of the $\mathrm{O} / \mathrm{H}$ radical pool has been developed in previous work. ${ }^{27}$ On the basis of reference calculations with a full reaction mechanism (see below), equations are derived in the present work to describe also the formation and consumption of relevant hydrocarbon radicals.

The combined model, i.e., the schemes for the $\mathrm{O} / \mathrm{H}$ and hydrocarbon radical pools and the reduced scheme for nitrogen chemistry, is tested by a comparison to reference calculations with a full mechanism, adopted from the work by Mendiara and Glarborg. ${ }^{20,23}$ Subsets of the full mechanism have been evaluated against a range of experimental data for oxidation of $\mathrm{HCN}^{34}$ and $\mathrm{NH}_{3}{ }^{19,23,35}$ and for reduction of NO. ${ }^{20}$ As documented in these references, the detailed mechanism provides a good description of volatile $\mathrm{N}$ oxidation and reburn-type chemistry over a wide range of conditions. For this reason, we find it appropriate to use modeling predictions with the detailed mechanism as reference calculations for the simplified schemes.

2.1. Prediction of Hydrocarbon Radicals. The elementary reactions taken into consideration for estimating concentrations of hydrocarbon radicals are listed in Table 2. It is mostly drawn from the skeletal mechanism of Glarborg et al. ${ }^{7}$ The $\mathrm{C}_{1}$ radicals needed in the reburning model are those belonging to the sequence $\mathrm{CH}_{3} \rightarrow \mathrm{CH}_{2}(\mathrm{~s}) \rightarrow \mathrm{CH}_{2} \rightarrow \mathrm{CH} \rightarrow \mathrm{C}$. These radicals can all be assumed to be in steady state. The equations describing the steady-state approximations are mostly drawn from Glarborg et al. ${ }^{7}$ The methyl radical, $\mathrm{CH}_{3}$, is involved in reactions R5-R16 in Table 2. However, because the influence of reactions $\mathrm{R} 11, \mathrm{R} 12$, and $\mathrm{R} 14$ on $\mathrm{CH}_{3}$ is minor, these steps are omitted from the steady-state approximation describing $\mathrm{CH}_{3}$. Furthermore, the reverse of reaction R17 is neglected (Table 3 ). The set of equations to determine $\mathrm{CH}_{3}$ then becomes (eq 1)

$$
\begin{aligned}
& D_{\mathrm{CH}_{3}}=k_{\mathrm{r}, \mathrm{R} 5}\left[\mathrm{H}_{2}\right]+k_{\mathrm{r}, \mathrm{R} 7}\left[\mathrm{H}_{2} \mathrm{O}\right]+\left(k_{\mathrm{f}, \mathrm{R} 8}+k_{\mathrm{f}, \mathrm{R} 9}\right)\left[\mathrm{O}_{2}\right] \\
& +k_{\mathrm{f}, \mathrm{R} 10}[\mathrm{H}]+k_{\mathrm{f}, \mathrm{R} 13}[\mathrm{O}]+\left(k_{\mathrm{f}, \mathrm{R} 15}+k_{\mathrm{f}, \mathrm{R} 16)[\mathrm{OH}]}\right. \\
& {\left[\mathrm{CH}_{3}\right]=\frac{\left[\mathrm{CH}_{4}\right]\left(k_{\mathrm{f}, \mathrm{R} 5}[\mathrm{H}]+k_{\mathrm{f}, \mathrm{R} 6}[\mathrm{O}]+k_{\mathrm{f}, \mathrm{R} 7}[\mathrm{OH}]\right)}{D_{\mathrm{CH}_{3}}}}
\end{aligned}
$$

(30) Suuberg, E. M.; Peters, W. A.; Howard, J. B. Ind. Eng. Chem. Process Des. Dev. 1978, 17, 37-46.

(31) Niksa, S.; Cho, S. Energy Fuels 1996, 10, 463-473.

(32) Neoh, K. G.; Gannon, R. E. Fuel 1984, 63, 1347-1352.

\begin{tabular}{|c|c|c|c|c|}
\hline number & reaction & $A(\mathrm{~cm}, \mathrm{~mol}, \mathrm{~s})$ & $b$ & $E(\mathrm{cal} / \mathrm{mol})$ \\
\hline R1 & $\mathrm{C}_{2} \mathrm{H}_{2}+\mathrm{O} \rightarrow \mathrm{CH}_{2}+\mathrm{CO}$ & $1.4 \times 10^{7}$ & 2.00 & 1900 \\
\hline $\mathrm{R} 2$ & $\mathrm{C}_{2} \mathrm{H}_{2}+\mathrm{O} \rightarrow \mathrm{HCCO}+\mathrm{H}$ & $6.1 \times 10^{6}$ & 2.00 & 1900 \\
\hline R3 & $\mathrm{HCCO}+\mathrm{H} \rightarrow \mathrm{CH}_{2}(\mathrm{~s})+\mathrm{CO}$ & $1.5 \times 10^{14}$ & 0.00 & 0 \\
\hline R4 & $\mathrm{HCCO}+\mathrm{O}_{2} \rightarrow \mathrm{CO}_{2}+\mathrm{CO}+\mathrm{H}$ & $4.9 \times 10^{12}$ & -0.142 & 1150 \\
\hline R5 & $\mathrm{CH}_{4}+\mathrm{H} \leftrightharpoons \mathrm{CH}_{3}+\mathrm{H}_{2}$ & $4.1 \times 10^{3}$ & 3.156 & 8755 \\
\hline R6 & $\mathrm{CH}_{4}+\mathrm{O} \rightarrow \mathrm{CH}_{3}+\mathrm{OH}$ & $4.4 \times 10^{5}$ & 2.50 & 6577 \\
\hline R7 & $\mathrm{CH}_{4}+\mathrm{OH} \leftrightharpoons \mathrm{CH}_{3}+\mathrm{H}_{2} \mathrm{O}$ & $1.0 \times 10^{6}$ & 2.182 & 2506 \\
\hline $\mathrm{R} 8$ & $\mathrm{CH}_{3}+\mathrm{O}_{2} \rightarrow \mathrm{CH}_{3} \mathrm{O}+\mathrm{O}$ & $7.5 \times 10^{12}$ & 0.00 & 28297 \\
\hline R9 & $\mathrm{CH}_{3}+\mathrm{O}_{2} \rightarrow \mathrm{CH}_{2} \mathrm{O}+\mathrm{OH}$ & $1.9 \times 10^{11}$ & 0.00 & 9842 \\
\hline R10 & $\mathrm{CH}_{3}+\mathrm{H} \rightarrow \mathrm{CH}_{4}$ & $2.6 \times 10^{28}$ & -5.10 & -2630 \\
\hline R11 & $\mathrm{CH}_{3}+\mathrm{H} \leftrightharpoons \mathrm{CH}_{2}+\mathrm{H}_{2}$ & $9.0 \times 10^{13}$ & 0.00 & 15100 \\
\hline $\mathrm{R} 12$ & $\mathrm{CH}_{3}+\mathrm{H} \leftrightharpoons \mathrm{CH}_{2}(\mathrm{~s})+\mathrm{H}_{2}$ & $1.2 \times 10^{17}$ & -0.80 & 16483 \\
\hline R13 & $\mathrm{CH}_{3}+\mathrm{O} \rightarrow \mathrm{CH}_{2} \mathrm{O}+\mathrm{H}$ & $9 \times 10^{13}$ & 0.00 & 0 \\
\hline R14 & $\mathrm{CH}_{3}+\mathrm{OH} \leftrightharpoons \mathrm{CH}_{2}+\mathrm{H}_{2} \mathrm{O}$ & $1 \times 10^{3}$ & 3.00 & 2780 \\
\hline R15 & $\mathrm{CH}_{3}+\mathrm{OH} \leftrightharpoons \mathrm{CH}$ & $6.9 \times 10^{14}$ & -0.4884 & 0 \\
\hline R16 & $\mathrm{CH}_{3}+\mathrm{OHC} \leftrightharpoons \mathrm{H}_{2} \mathrm{OH}+\mathrm{H}$ & $5.4 \times 10^{10}$ & 1.00 & 3554 \\
\hline R17 & $\mathrm{CH}_{2}+\mathrm{H} \rightarrow \mathrm{CH}_{3}$ & $5.3 \times 10^{24}$ & -3.90 & 829 \\
\hline R18 & $\mathrm{CH}_{2}+\mathrm{H} \leftrightharpoons \mathrm{CH}+\mathrm{H}_{2}$ & $1.2 \times 10^{14}$ & 0.00 & 0 \\
\hline R19 & $\mathrm{CH}_{2}+\mathrm{O} \rightarrow \mathrm{CO}+\mathrm{H}+\mathrm{H}$ & $2 \times 10^{14}$ & 0.00 & 536 \\
\hline $\mathrm{R} 20$ & $\mathrm{CH}_{2}+\mathrm{O} \rightarrow \mathrm{CO}+\mathrm{H}_{2}$ & $8.0 \times 10^{13}$ & 0.00 & 536 \\
\hline $\mathrm{R} 21$ & $\mathrm{CH}_{2}+\mathrm{OH} \rightarrow \mathrm{CH}_{2} \mathrm{O}+\mathrm{H}$ & $2.8 \times 10^{13}$ & 0.1228 & -161 \\
\hline R22 & $\mathrm{CH}_{2}+\mathrm{OH} \leftrightharpoons \mathrm{CH}+\mathrm{H}_{2} \mathrm{O}$ & $8.6 \times 10^{5}$ & 2.019 & 6776 \\
\hline $\mathrm{R} 23$ & $\mathrm{CH}_{2}+\mathrm{O}_{2} \rightarrow \mathrm{CH}_{2} \mathrm{O}+\mathrm{O}$ & $2.9 \times 10^{11}$ & 0.00 & 0 \\
\hline R24 & $\mathrm{CH}_{2}+\mathrm{O}_{2} \rightarrow \mathrm{CO}_{2}+\mathrm{H}_{2}$ & $1.5 \times 10^{12}$ & 0.00 & 0 \\
\hline $\mathrm{R} 25$ & $\mathrm{CH}_{2}+\mathrm{CO}_{2} \rightarrow \mathrm{CO}+\mathrm{CH}_{2} \mathrm{O}$ & $1.0 \times 10^{11}$ & 0.00 & 1000 \\
\hline \multirow{2}{*}{ R26 } & $\mathrm{CH}_{2}(\mathrm{~s})+\mathrm{M} \leftrightharpoons \mathrm{CH}_{2}+\mathrm{M}$ & $1.0 \times 10^{13}$ & 0.00 & 0 \\
\hline & \multicolumn{4}{|c|}{ third body enhancements: $\mathrm{H}_{2}=20, \mathrm{O}_{2}=3.1$, and $\mathrm{H}_{2} \mathrm{O}=3$} \\
\hline R27 & $\mathrm{CH}_{2}(\mathrm{~s})+\mathrm{N}_{2} \leftrightharpoons \mathrm{CH}_{2}+\mathrm{N}_{2}$ & $1.3 \times 10^{13}$ & 0.00 & 430 \\
\hline R28 & $\mathrm{CH}_{2}(\mathrm{~s})+\mathrm{O}_{2} \rightarrow \mathrm{CO}+\mathrm{OH}+\mathrm{H}$ & $3.1 \times 10^{13}$ & 0.00 & 0 \\
\hline R29 & $\mathrm{CH}_{2}(\mathrm{~s})+\mathrm{CO}_{2} \rightarrow \mathrm{CH}_{2} \mathrm{O}+\mathrm{CO}$ & $1.1 \times 10^{13}$ & 0.00 & 0 \\
\hline R30 & $\mathrm{CH}+\mathrm{H} \leftrightharpoons \mathrm{C}+\mathrm{H}_{2}$ & $1.5 \times 10^{14}$ & 0.00 & 0 \\
\hline R31 & $\mathrm{CH}+\mathrm{O} \rightarrow \mathrm{CO}+\mathrm{H}$ & $5.7 \times 10^{13}$ & 0.00 & 0 \\
\hline R32 & $\mathrm{CH}+\mathrm{OH} \rightarrow \mathrm{HCO}+\mathrm{H}$ & $3.0 \times 10^{13}$ & 0.00 & 0 \\
\hline R33 & $\mathrm{CH}+\mathrm{OH} \leftrightharpoons \mathrm{C}+\mathrm{H}_{2} \mathrm{O}$ & $4.0 \times 10^{7}$ & 2.00 & 3000 \\
\hline R34 & $\mathrm{CH}+\mathrm{O}_{2} \rightarrow \mathrm{HCO}+\mathrm{O}$ & $3.3 \times 10^{13}$ & 0.00 & 0 \\
\hline R35 & $\mathrm{CH}+\mathrm{H}_{2} \mathrm{O} \rightarrow \mathrm{CH}_{2} \mathrm{O}+\mathrm{H}$ & $5.7 \times 10^{12}$ & 0.00 & -755 \\
\hline R36 & $\mathrm{CH}+\mathrm{CO}_{2} \rightarrow \mathrm{HCO}+\mathrm{CO}$ & $8.8 \times 10^{6}$ & 1.75 & -1040 \\
\hline R37 & $\mathrm{C}+\mathrm{OH} \rightarrow \mathrm{CO}+\mathrm{H}$ & $5.0 \times 1$ & 0.00 & 0 \\
\hline R38 & $\mathrm{C}+\mathrm{O}_{2} \rightarrow \mathrm{CO}+\mathrm{O}$ & $2.0 \times 10^{13}$ & 0.00 & 0 \\
\hline
\end{tabular}

Table 2. Reactions Considered for Estimating Hydrocarbon Radical Concentrations $^{a}$

${ }^{a}$ Rate constant expressed as $k=A T^{b} \exp (-E /(R T))$.

Table 3. Reverse Reaction Rates for Reversible Reactions ${ }^{a}$

\begin{tabular}{llllr}
\hline number & \multicolumn{1}{c}{ reaction } & $A(\mathrm{~cm}, \mathrm{~mol}, \mathrm{~s})$ & $b$ & $E(\mathrm{cal} / \mathrm{mol})$ \\
\hline $\mathrm{R} 5$ & $\mathrm{CH}_{4}+\mathrm{H} \leftrightharpoons \mathrm{CH}_{3}+\mathrm{H}_{2}$ & $1.30 \times 10^{-1}$ & 4 & 5540 \\
R7 & $\mathrm{CH}_{4}+\mathrm{OH} \leftrightharpoons \mathrm{CH}_{3}+\mathrm{H}_{2} \mathrm{O}$ & $1.63 \times 10^{5}$ & 2.18 & 16429 \\
$\mathrm{R} 11$ & $\mathrm{CH}_{3}+\mathrm{H} \leftrightharpoons \mathrm{CH}_{2}+\mathrm{H}_{2}$ & $3.90 \times 10^{9}$ & 1 & 6930 \\
$\mathrm{R} 12$ & $\mathrm{CH}_{3}+\mathrm{H} \leftrightharpoons \mathrm{CH}_{2}(\mathrm{~s})+\mathrm{H}_{2}$ & $7.20 \times 10^{13}$ & 0 & 0 \\
R14 & $\mathrm{CH}_{3}+\mathrm{OH} \leftrightharpoons \mathrm{CH}_{2}+\mathrm{H}_{2} \mathrm{O}$ & $3.18 \times 10^{1}$ & 3.4 & 11018 \\
R15 & $\mathrm{CH}_{3}+\mathrm{OH} \leftrightharpoons \mathrm{CH}_{2}(\mathrm{~s})+\mathrm{H}_{2} \mathrm{O}$ & $2.42 \times 10^{13}$ & 0 & -951 \\
R16 & $\mathrm{CH}_{3}+\mathrm{OH} \leftrightharpoons \mathrm{CH}_{2} \mathrm{OH}+\mathrm{H}$ & $1.80 \times 10^{14}$ & 0.16 & 111 \\
R18 & $\mathrm{CH}_{2}+\mathrm{H} \leftrightharpoons \mathrm{CH}+\mathrm{H}_{2}$ & $8.42 \times 10^{13}$ & 0 & 2950 \\
R22 & $\mathrm{CH}_{2}+\mathrm{OHCH}+\mathrm{H}_{2} \mathrm{O}$ & $5.58 \times 10^{13}$ & 0 & 30420 \\
R26 & $\mathrm{CH}_{2}(\mathrm{~s})+\mathrm{M} \leftrightharpoons \mathrm{CH}_{2}+\mathrm{M}$ & $3.99 \times 10^{12}$ & 0 & 8919 \\
\multicolumn{5}{c}{ third body enhancements: $\mathrm{H}_{2}=20, \mathrm{O}_{2}=3.1$, and $\mathrm{H}_{2} \mathrm{O}=3$} \\
R27 & $\mathrm{CH}_{2}(\mathrm{~s})+\mathrm{N}=\mathrm{CH}_{2}+\mathrm{N}_{2}$ & $4.90 \times 10^{12}$ & 0 & 9369 \\
R30 & $\mathrm{CH}+\mathrm{H} \leftrightharpoons \mathrm{C}+\mathrm{H}_{2}$ & $5.55 \times 10^{14}$ & 0 & 24065 \\
R33 & $\mathrm{CH}+\mathrm{OH} \leftrightharpoons \mathrm{C}+\mathrm{H}_{2} \mathrm{O}$ & $6.62 \times 10^{8}$ & 2 & 41667
\end{tabular}

${ }^{a}$ Numbering is the same as in Table 2.

Here, $k_{\mathrm{f}}$ and $k_{\mathrm{r}}$ denote forward and reverse rate constants, respectively. The steady-state approximations for $\mathrm{CH}_{2}$ and $\mathrm{CH}_{2}$ (s) are solved simultaneously, because these radicals are linked through reactions R26 and R27. For simplification, reactions $\mathrm{R} 18$ and $\mathrm{R} 22$ are assumed to be irreversible in the determination of $\mathrm{CH}_{2}$; the contribution from $\mathrm{CH}$ by the reverse reactions is expected to be small. The equations for $\mathrm{CH}_{2}$ 
are as follows:

$$
\begin{aligned}
D_{\mathrm{CH}_{2}}= & k_{\mathrm{r}, \mathrm{R} 11}\left[\mathrm{H}_{2}\right]+k_{\mathrm{r}, \mathrm{R} 14}\left[\mathrm{H}_{2} \mathrm{O}\right]+\left(k_{\mathrm{f}, \mathrm{R} 17}+k_{\mathrm{f}, \mathrm{R} 18)[\mathrm{H}]}\right. \\
& +\left(k_{\mathrm{f}, \mathrm{R} 19}+k_{\mathrm{f}, \mathrm{R} 20}\right)[\mathrm{O}]+\left(k_{\mathrm{f}, \mathrm{R} 21}+k_{\mathrm{f}, \mathrm{R} 22}\right)[\mathrm{OH}] \\
+\left(k_{\mathrm{f}, \mathrm{R} 23}+k_{\mathrm{f}, \mathrm{R} 24}\right)\left[\mathrm{O}_{2}\right]+k_{\mathrm{f}, \mathrm{R} 25}\left[\mathrm{CO}_{2}\right]+k_{\mathrm{r}, \mathrm{R} 26}[\mathrm{M}]+k_{\mathrm{r}, \mathrm{R} 27}\left[\mathrm{~N}_{2}\right] & \\
N_{1, \mathrm{CH}_{2}}= & \frac{k_{\mathrm{f}, \mathrm{R} 1}\left[\mathrm{C}_{2} \mathrm{H}_{2}\right][\mathrm{O}]+\left(k_{\mathrm{f}, \mathrm{R} 11}[\mathrm{H}]+k_{\mathrm{f}, \mathrm{R} 14}[\mathrm{OH}]\right)\left[\mathrm{CH}_{3}\right]}{D_{\mathrm{CH}_{2}}} \\
N_{2, \mathrm{CH}_{2}}= & \frac{k_{\mathrm{f}, \mathrm{R} 26}[\mathrm{M}]+k_{\mathrm{f}, \mathrm{R} 27}\left[\mathrm{~N}_{2}\right]}{D_{\mathrm{CH}_{2}}} \\
{\left[\mathrm{CH}_{2}\right]=} & N_{1, \mathrm{CH}_{2}}+N_{2, \mathrm{CH}_{2}}\left[\mathrm{CH}_{2}(\mathrm{~s})\right]
\end{aligned}
$$

The equations for $\mathrm{CH}_{2}$ (s) are as follows:

$$
\begin{aligned}
D_{\mathrm{CH}_{2}(\mathrm{~s})}= & k_{\mathrm{r}, \mathrm{R} 12}\left[\mathrm{H}_{2}\right]+k_{\mathrm{r}, \mathrm{R} 15}\left[\mathrm{H}_{2} \mathrm{O}\right]+k_{\mathrm{f}, \mathrm{R} 26}[\mathrm{M}]+k_{\mathrm{f}, \mathrm{R} 27}\left[\mathrm{~N}_{2}\right] \\
& +k_{\mathrm{f}, \mathrm{R} 28}\left[\mathrm{O}_{2}\right]+k_{\mathrm{f}, \mathrm{R} 29}\left[\mathrm{CO}_{2}\right] \\
N_{1, \mathrm{CH}_{2}(\mathrm{~s})}= & \frac{k_{\mathrm{f}, \mathrm{R} 3}[\mathrm{HCCO}][\mathrm{H}]+\left(k_{\mathrm{f}, \mathrm{R} 12}[\mathrm{H}]+k_{\mathrm{f}, \mathrm{R} 15}[\mathrm{OH}]\right)\left[\mathrm{CH}_{3}\right]}{D_{\mathrm{CH}_{2}(\mathrm{~s})}} \\
N_{2, \mathrm{CH}_{2}(\mathrm{~s})}= & \frac{k_{\mathrm{r}, \mathrm{R} 26}[\mathrm{M}]+k_{\mathrm{r}, \mathrm{R} 27}\left[\mathrm{~N}_{2}\right]}{D_{\mathrm{CH}_{2}(\mathrm{~s})}} \\
{\left[\mathrm{CH}_{2}(\mathrm{~s})\right]=} & N_{1, \mathrm{CH}_{2}(\mathrm{~s})}+N_{2, \mathrm{CH}_{2}(\mathrm{~s})}\left[\mathrm{CH}_{2}\right]
\end{aligned}
$$

The concentration of $\mathrm{CH}_{2}$ can, by simultaneously solving eqs 2 and 3 , be calculated by eq 4 .

$$
\left[\mathrm{CH}_{2}\right]=\frac{N_{1, \mathrm{CH}_{2}}+N_{2, \mathrm{CH}_{2}} N_{1, \mathrm{CH}_{2}(\mathrm{~s})}}{1-N_{2, \mathrm{CH}_{2}} N_{2, \mathrm{CH}_{2}(\mathrm{~s})}}
$$

The concentrations of the radicals $\mathrm{CH}$ and $\mathrm{C}$ are also solved simultaneously because they are linked through reactions R30 and $\mathrm{R} 33$. The equation for determining the $\mathrm{CH}$ radical concentration is as follows:

\begin{tabular}{|c|c|c|c|}
\hline \multirow[b]{2}{*}{ fuel number } & \multicolumn{3}{|c|}{$\varepsilon=A_{\varepsilon} T+B_{\varepsilon}$} \\
\hline & \multicolumn{2}{|c|}{$A_{\varepsilon}$} & $B_{\varepsilon}$ \\
\hline 1 & \multicolumn{2}{|c|}{$4.43 \times 10^{-4}$} & $-4.71 \times 10^{-1}$ \\
\hline 2 & \multicolumn{2}{|c|}{$4.78 \times 10^{-4}$} & $-4.74 \times 10^{-1}$ \\
\hline 3 & \multicolumn{2}{|c|}{$5.25 \times 10^{-4}$} & $-4.44 \times 10^{-1}$ \\
\hline \multirow[t]{2}{*}{4} & \multicolumn{2}{|c|}{$6.49 \times 10^{-5}$} & $-7.09 \times 10^{-2}$ \\
\hline & \multicolumn{3}{|c|}{$\kappa=A_{\kappa} T^{2}+B_{\kappa} T+C_{\kappa}$} \\
\hline fuel number & $A_{\kappa}$ & $B_{\kappa}$ & $C_{\kappa}$ \\
\hline 1 & $-4.38 \times 10^{-7}$ & $1.77 \times 10^{-3}$ & -1.96 \\
\hline 2 & $-4.40 \times 10^{-7}$ & $1.79 \times 10^{-3}$ & -1.20 \\
\hline 3 & $-4.40 \times 10^{-7}$ & $1.80 \times 10^{-3}$ & -2.01 \\
\hline 4 & $-3.93 \times 10^{-7}$ & $1.71 \times 10^{-3}$ & -1.95 \\
\hline
\end{tabular}

$$
\begin{aligned}
D_{\mathrm{CH}}= & k_{\mathrm{r}, \mathrm{R} 18}\left[\mathrm{H}_{2}\right]+k_{\mathrm{r}, \mathrm{R} 22}\left[\mathrm{H}_{2} \mathrm{O}\right]+k_{\mathrm{f}, \mathrm{R} 30}[\mathrm{H}]+k_{\mathrm{f}, \mathrm{R} 31}[\mathrm{O}] \\
& +\left(k_{\mathrm{f}, \mathrm{R} 32}+k_{\mathrm{f}, \mathrm{R} 33}\right)[\mathrm{OH}]+k_{\mathrm{f}, \mathrm{R} 34}\left[\mathrm{O}_{2}\right] \\
& +k_{\mathrm{f}, \mathrm{R} 35}\left[\mathrm{H}_{2} \mathrm{O}\right]+k_{\mathrm{f}, \mathrm{R} 36}\left[\mathrm{CO}_{2}\right] \\
N_{1, \mathrm{CH}}= & \frac{\left[\mathrm{CH}_{2}\right]\left(k_{\mathrm{f}, \mathrm{R} 18}[\mathrm{H}]+k_{\mathrm{f}, \mathrm{R} 22}[\mathrm{OH}]\right)}{D_{\mathrm{CH}}} \\
N_{2, \mathrm{CH}}= & \frac{k_{\mathrm{r}, \mathrm{R} 30}\left[\mathrm{H}_{2}\right]+k_{\mathrm{r}, \mathrm{R} 33}\left[\mathrm{H}_{2} \mathrm{O}\right]}{D_{\mathrm{CH}}} \\
{[\mathrm{CH}]=} & N_{1, \mathrm{CH}}+N_{2, \mathrm{CH}}[\mathrm{C}]
\end{aligned}
$$

The $\mathrm{C}$ radical concentration is calculated by eq 6 .

$$
\begin{aligned}
& D_{\mathrm{C}}=k_{\mathrm{r}, \mathrm{R} 30}\left[\mathrm{H}_{2}\right]+k_{\mathrm{r}, \mathrm{R} 33}\left[\mathrm{H}_{2} \mathrm{O}\right]+k_{\mathrm{f}, \mathrm{R} 37}[\mathrm{OH}]+k_{\mathrm{f}, \mathrm{R} 38}\left[\mathrm{O}_{2}\right] \\
& N_{\mathrm{C}}=\frac{k_{\mathrm{f}, \mathrm{R} 30}[\mathrm{H}]+k_{\mathrm{f}, \mathrm{R} 33}[\mathrm{OH}]}{D_{\mathrm{C}}} \\
& {[\mathrm{C}]=N_{\mathrm{C}}[\mathrm{CH}]}
\end{aligned}
$$

(33) Vilas, E.; Skifter, U.; Jensen, A. D.; López, C.; Maier, J.; Glarborg, P. Energy Fuels 2004, 18, 1442-1450.

(34) Dagaut, P.; Glarborg, P.; Alzueta, M. U. Prog. Energy Combust. Sci. 2008, 34, 1-46.

(35) Tian, Z.; Li, Y.; Zhang, L.; Glarborg, P.; Qi, F. Combust. Flame 2009, 156, 1413-1426.
Table 4. Values for the Calculation of $Q_{3}$

When eqs 5 and 6 are solved simultaneously, the $\mathrm{CH}$ concentration can be determined by eq 7 .

$$
[\mathrm{CH}]=\frac{N_{1, \mathrm{CH}}}{1-N_{2, \mathrm{CH}} N_{\mathrm{C}}}
$$

The $\mathrm{C}_{2}$ chemistry added in the present work to the scheme of Glarborg et al. ${ }^{7}$ aims at estimating the concentration of $\mathrm{HCCO}$, which is formed from $\mathrm{C}_{2} \mathrm{H}_{2}$. Inclusion of an analytically reduced submodel for the $\mathrm{C}_{2}$ chemistry would complicate the model significantly. Instead, semi-empirical correlations, similar to those reported for the $\mathrm{O} / \mathrm{H}$ radical pool, ${ }^{27}$ were developed. These were based on reference calculations with the full mechanism, assuming isothermal plug-flow conditions and representing the fuel by the different volatile compositions given in Table 1. It was found that the formation and consumption of $\mathrm{C}_{2} \mathrm{H}_{2}$ during combustion show similarities to the profile predicted for $\mathrm{CH}_{3}$. Accordingly, $\mathrm{C}_{2} \mathrm{H}_{2}$ may be described by eq 8 .

$$
\left[\mathrm{C}_{2} \mathrm{H}_{2}\right]=Q_{3}\left[\mathrm{CH}_{3}\right]
$$

Values of $Q_{3}$ were then determined from the peak concentrations of $\mathrm{C}_{2} \mathrm{H}_{2}$ and $\mathrm{CH}_{3}$ predicted by the detailed mechanism for temperatures in the range of 1200-2000 K, inlet oxygen concentrations of $1-21 \mathrm{vol} \%$, and values of $\lambda$ in the range of $0.6-2.0$. Various functional forms describing $Q_{3}$ as a function of combustion conditions were tested. The following simple correlation was found to be satisfactory for the three coals:

$$
Q_{3}=\frac{1}{\sqrt{\lambda}} \varepsilon(T)\left[\mathrm{O}_{2}\right]_{\mathrm{in}}^{\kappa(T)}
$$

For biomass, where the volatiles were not assumed to contain $\mathrm{C}_{2} \mathrm{H}_{4}$ (Table 1), $\mathrm{C}_{2}$ hydrocarbons are formed only through recombination of $\mathrm{C}_{1}$ compounds. Here, the correlation is

$$
Q_{3}=\frac{1}{\lambda^{2}} \varepsilon(T)\left[\mathrm{O}_{2}\right]_{\text {in }}^{\kappa(T)}
$$

The constants, $\varepsilon$ and $\kappa$, are functions of the temperature and, furthermore, dependent upon the coal type. These can be calculated according to the correlations in Table 4.

The prediction of HCCO is obtained by setting up a steadystate balance involving reactions $\mathrm{R} 2-\mathrm{R} 4$ in Table 2 . The HCCO concentration is thus calculated by eq 11 .

$$
[\mathrm{HCCO}]=\frac{k_{\mathrm{f}, 2}[\mathrm{O}]\left[\mathrm{C}_{2} \mathrm{H}_{2}\right]}{k_{\mathrm{f}, 3}[\mathrm{H}]+k_{\mathrm{f}, 4}\left[\mathrm{O}_{2}\right]}
$$

2.2. $\mathbf{N}$ Chemistry Scheme. The scheme developed in this work describes reburn-type chemistry along with oxidation of $\mathrm{NH}_{3}$ and HCN. The basis of the model is the scheme of Pedersen et al., ${ }^{8}$ which describes well the $\mathrm{HCN}$ and $\mathrm{NH}_{3}$ oxidation chemistry. ${ }^{27}$ The Pedersen scheme draws on reactions N1-N36 in Table 5. This scheme was then extended with eight 
Table 5. Reactions Considered for Estimating the Nitrogen Chemistry $^{a}$

\begin{tabular}{|c|c|c|c|c|}
\hline number & reaction & $A(\mathrm{~cm}, \mathrm{~mol}, \mathrm{~s})$ & $b$ & $E(\mathrm{cal} / \mathrm{mol})$ \\
\hline N1 & $\mathrm{CN}+\mathrm{H}_{2} \rightleftharpoons \mathrm{HCN}+\mathrm{H}$ & $3.60 \times 10^{8}$ & 1.55 & 3000 \\
\hline $\mathrm{N} 2$ & $\mathrm{HCN}+\mathrm{O} \rightarrow \mathrm{NCO}+\mathrm{H}$ & $1.40 \times 10^{4}$ & 2.64 & 4980 \\
\hline N3 & $\mathrm{HCN}+\mathrm{O} \rightarrow \mathrm{NH}+\mathrm{CO}$ & $3.50 \times 10^{3}$ & 2.64 & 4980 \\
\hline $\mathrm{N} 4$ & $\mathrm{CN}+\mathrm{H}_{2} \mathrm{O} \rightleftharpoons \mathrm{HCN}+\mathrm{OH}$ & $8.00 \times 10^{12}$ & 0.00 & 7450 \\
\hline N5 & $\mathrm{HCN}+\mathrm{OH} \rightleftharpoons \mathrm{HOCN}+\mathrm{H}$ & $5.90 \times 10^{4}$ & 2.40 & 12500 \\
\hline N6 & $\mathrm{HOCN}+\mathrm{H} \rightarrow \mathrm{HNCO}+\mathrm{H}$ & $2.00 \times 10^{7}$ & 2.00 & 2000 \\
\hline N7 & $\mathrm{HOCN}+\mathrm{O} \rightarrow \mathrm{NCO}+\mathrm{OH}$ & $1.50 \times 10^{4}$ & 2.64 & 4000 \\
\hline N8 & $\mathrm{HOCN}+\mathrm{OH} \rightarrow \mathrm{NCO}+\mathrm{H}_{2} \mathrm{O}$ & $6.40 \times 10^{5}$ & 2.00 & 2560 \\
\hline N9 & $\mathrm{CN}+\mathrm{OH} \rightarrow \mathrm{NCO}+\mathrm{H}$ & $6.00 \times 10^{13}$ & 0.00 & 0 \\
\hline N10 & $\mathrm{CN}+\mathrm{O}_{2} \rightarrow \mathrm{NCO}+\mathrm{O}$ & $7.50 \times 10^{12}$ & 0.00 & -389 \\
\hline N11 & $\mathrm{HNCO}+\mathrm{OH} \rightleftharpoons \mathrm{NCO}+\mathrm{H}_{2} \mathrm{O}$ & $6.40 \times 10^{5}$ & 2.00 & 2560 \\
\hline N12 & $\mathrm{NCO}+\mathrm{H}_{2} \rightleftharpoons \mathrm{HNCO}+\mathrm{H}$ & $7.60 \times 10^{2}$ & 3.00 & 4000 \\
\hline N13 & $\mathrm{NCO}+\mathrm{M} \rightarrow \mathrm{N}+\mathrm{CO}+\mathrm{M}$ & $3.10 \times 10^{16}$ & -0.50 & 48000 \\
\hline \multicolumn{5}{|c|}{ third body enhancement: $\mathrm{N}_{2}=1.5$} \\
\hline N14 & $\mathrm{NCO}+\mathrm{H} \rightarrow \mathrm{CO}+\mathrm{NH}$ & $5.00 \times 10^{13}$ & 0.00 & 0 \\
\hline N15 & $\mathrm{NCO}+\mathrm{O} \rightarrow \mathrm{NO}+\mathrm{CO}$ & $4.70 \times 10^{13}$ & 0.00 & 0 \\
\hline N16 & $\mathrm{NCO}+\mathrm{NO} \rightarrow \mathrm{N}_{2}+\mathrm{CO}_{2}$ & $1.40 \times 10^{18}$ & -1.73 & 763 \\
\hline N17 & $\mathrm{HNCO}+\mathrm{M} \rightarrow \mathrm{CO}+\mathrm{NH}$ & $1.10 \times 10^{16}$ & 0.00 & 86000 \\
\hline \multicolumn{5}{|c|}{ third body enhancement: $\mathrm{N}_{2}=1.5$} \\
\hline N18 & $\mathrm{HNCO}+\mathrm{O}_{2} \rightarrow \mathrm{H}+\mathrm{NO}+\mathrm{CO}_{2}$ & $1.00 \times 10^{12}$ & 0.00 & 35000 \\
\hline N19 & $\mathrm{HNCO}+\mathrm{H} \rightarrow \mathrm{NH}_{2}+\mathrm{CO}$ & $2.20 \times 10^{7}$ & 1.70 & 3800 \\
\hline N20 & $\mathrm{HNCO}+\mathrm{O} \rightarrow \mathrm{NH}+\mathrm{CO}_{2}$ & $9.60 \times 10^{7}$ & 1.41 & 8520 \\
\hline $\mathrm{N} 21$ & $\mathrm{NH}_{3}+\mathrm{M} \rightleftharpoons \mathrm{NH}_{2}+\mathrm{H}+\mathrm{M}$ & $2.20 \times 10^{16}$ & 0.00 & 93470 \\
\hline $\mathrm{N} 22$ & $\mathrm{NH}_{3}+\mathrm{OH} \rightleftharpoons \mathrm{NH}_{2}+\mathrm{H}_{2} \mathrm{O}$ & $2.00 \times 10^{6}$ & 2.04 & 566 \\
\hline N23 & $\mathrm{NH}_{3}+\mathrm{H} \rightleftharpoons \mathrm{NH}_{2}+\mathrm{H}_{2}$ & $6.40 \times 10^{5}$ & 2.39 & 10171 \\
\hline N24 & $\mathrm{NH}_{2}+\mathrm{H} \rightleftharpoons \mathrm{NH}+\mathrm{H}_{2}$ & $4.00 \times 10^{13}$ & 0.00 & 3650 \\
\hline N25 & $\mathrm{NH}_{2}+\mathrm{OH} \rightleftharpoons \mathrm{NH}+\mathrm{H}_{2} \mathrm{O}$ & $4.00 \times 10^{6}$ & 2.00 & 1000 \\
\hline N26 & $\mathrm{NH}_{2}+\mathrm{NO} \rightarrow \mathrm{N}_{2}+\mathrm{H}_{2} \mathrm{O}$ & $1.30 \times 10^{16}$ & -1.25 & 0 \\
\hline $\mathrm{N} 27$ & $\mathrm{NH}+\mathrm{O}_{2} \rightarrow \mathrm{H}+\mathrm{NO}+\mathrm{O}$ & $4.60 \times 10^{5}$ & 2.00 & 6500 \\
\hline $\mathrm{N} 28$ & $\mathrm{NH}+\mathrm{O}_{2} \rightarrow \mathrm{NO}+\mathrm{OH}$ & $1.30 \times 10^{6}$ & 1.50 & 100 \\
\hline N29 & $\mathrm{NH}+\mathrm{H} \rightarrow \mathrm{N}+\mathrm{H}_{2}$ & $3.00 \times 10^{13}$ & 0.00 & 0 \\
\hline N30 & $\mathrm{NH}+\mathrm{O} \rightarrow \mathrm{NO}+\mathrm{H}$ & $9.20 \times 10^{13}$ & 0.00 & 0 \\
\hline N31 & $\mathrm{NH}+\mathrm{OH} \rightarrow \mathrm{H}+\mathrm{NO}+\mathrm{H}$ & $2.00 \times 10^{13}$ & 0.00 & 0 \\
\hline N32 & $\mathrm{NH}+\mathrm{OH} \rightleftharpoons \mathrm{N}+\mathrm{H}_{2} \mathrm{O}$ & $5.00 \times 10^{11}$ & 0.50 & 2000 \\
\hline N33 & $\mathrm{NH}+\mathrm{NO} \rightarrow \mathrm{N}_{2}+\mathrm{O}+\mathrm{H}$ & $2.90 \times 10^{14}$ & -0.40 & 0 \\
\hline N34 & $\mathrm{N}+\mathrm{OH} \rightleftharpoons \mathrm{NO}+\mathrm{H}$ & $3.80 \times 10^{13}$ & 0.00 & 0 \\
\hline N35 & $\mathrm{N}+\mathrm{O}_{2} \rightleftharpoons \mathrm{NO}+\mathrm{O}$ & $6.40 \times 10^{9}$ & 1.00 & 6280 \\
\hline N36 & $\mathrm{N}+\mathrm{NO} \rightleftharpoons \mathrm{N}_{2}+\mathrm{O}$ & $3.30 \times 10^{12}$ & 0.30 & 0 \\
\hline N37 & $\mathrm{CH}+\mathrm{N}_{2} \rightarrow \mathrm{HCN}+\mathrm{N}$ & $3.70 \times 10^{7}$ & 1.42 & 20730 \\
\hline N38 & $\mathrm{C}+\mathrm{N}_{2} \rightarrow \mathrm{CN}+\mathrm{N}$ & $6.31 \times 10^{13}$ & 0.00 & 46000 \\
\hline N39 & $\mathrm{CH}_{2}+\mathrm{NO} \rightarrow \mathrm{NCO}+\mathrm{H}+\mathrm{H}$ & $8.98 \times 10^{7}$ & 1.66 & 13910 \\
\hline N40 & $\mathrm{CH}_{2}(\mathrm{~s})+\mathrm{NO} \rightarrow \mathrm{HCN}+\mathrm{OH}$ & $2.00 \times 10^{13}$ & 0.00 & 0 \\
\hline N41 & $\mathrm{CH}+\mathrm{NO} \rightarrow \mathrm{HCN}+\mathrm{O}$ & $7.90 \times 10^{13}$ & 0.00 & 0 \\
\hline N42 & $\mathrm{C}+\mathrm{NO} \rightarrow \mathrm{CN}+\mathrm{O}$ & $2.00 \times 10^{13}$ & 0.00 & 0 \\
\hline N43 & $\mathrm{C}+\mathrm{NO} \rightarrow \mathrm{N}+\mathrm{CO}$ & $2.80 \times 10^{13}$ & 0.00 & 0 \\
\hline N44 & $\mathrm{N}+\mathrm{CH}_{3} \rightarrow \mathrm{HCN}+\mathrm{H}+\mathrm{H}$ & $2.40 \times 10^{8}$ & 1.50 & -894 \\
\hline N45 & $\mathrm{HCCO}+\mathrm{NO} \rightarrow \mathrm{HCNO}+\mathrm{CO}$ & $5.90 \times 10^{12}$ & 0.09 & -457 \\
\hline N46 & $\mathrm{HCNO} \rightarrow \mathrm{HCN}+\mathrm{O}$ & $4.20 \times 10^{31}$ & -6.12 & 61210 \\
\hline N47 & $\mathrm{HCNO}+\mathrm{H} \rightarrow \mathrm{HCN}+\mathrm{OH}$ & $7.20 \times 10^{10}$ & 0.84 & 8612 \\
\hline
\end{tabular}

NO reduction reactions from the mechanism of Glarborg et al. ${ }^{7}$ (reactions N37-N44 in Table 5).

For the reburn reactions $(\mathrm{N} 37-\mathrm{N} 44)$, we have updated the rate constants according to the detailed mechanism by Mendiara and Glarborg. ${ }^{20,23}$ For several of these steps, more accurate kinetic parameters have become available since the publication of the reduced mechanism of Glarborg et al., ${ }^{7}$ but the changes had only a small impact on modeling predictions.

In the present work, the three reactions N45-N47 were added to the mechanism. These steps describe the reduction of NO by the reaction with the $\mathrm{HCCO}$ radical and the subsequent conversion of the HCNO intermediate. Removal of NO by $\mathrm{HCCO}$ was found to be most important in combustion of coals, which release significant amounts of $\mathrm{C}_{2}$ hydrocarbons with the volatiles.

The rates of production and consumption of the key nitrogen species are determined by the net rate of the reaction of selected
Table 6. Equilibrium Constants for Reversible Reactions ${ }^{a}$

\begin{tabular}{llllr}
\hline number & \multicolumn{1}{c}{ reaction } & \multicolumn{1}{c}{$A$} & \multicolumn{1}{c}{$b$} & $-E / R$ \\
\hline $\mathrm{N} 1$ & $\mathrm{CN}+\mathrm{H}_{2} \rightleftharpoons \mathrm{HCN}+\mathrm{H}$ & $1.2 \times 10^{-4}$ & 0.9545 & 10650 \\
$\mathrm{~N} 4$ & $\mathrm{CN}+\mathrm{H}_{2} \mathrm{O} \rightleftharpoons \mathrm{HCN}+\mathrm{OH}$ & $8.3 \times 10^{-3}$ & 0.6244 & 2599 \\
$\mathrm{~N} 5$ & $\mathrm{HCN}+\mathrm{OH} \rightleftharpoons \mathrm{HOCN}+\mathrm{H}$ & $2.2 \times 10^{-2}$ & 0.1689 & -3764 \\
$\mathrm{~N} 11$ & $\mathrm{HNCO}+\mathrm{OH} \rightleftharpoons \mathrm{NCO}+\mathrm{H}_{2} \mathrm{O}$ & $4.2 \times 10^{-1}$ & $-4.13 \times 10^{-2}$ & 3862 \\
$\mathrm{~N} 12$ & $\mathrm{NCO}+\mathrm{H} 2 \rightleftharpoons \mathrm{HNCO}+\mathrm{H}$ & $3.4 \times 10^{-2}$ & 0.3713 & 4190 \\
$\mathrm{~N} 21$ & $\mathrm{NH}_{3}+\mathrm{M} \rightleftharpoons \mathrm{NH}_{2}+\mathrm{H}+\mathrm{M}$ & $1.6 \times 10^{6}$ & 0.2232 & -55580 \\
$\mathrm{~N} 22$ & $\mathrm{NH}_{3}+\mathrm{OH} \rightleftharpoons \mathrm{NH}_{2}+\mathrm{H}_{2} \mathrm{O}$ & $9.6 \times 10^{1}$ & -0.4878 & 4984 \\
$\mathrm{~N} 23$ & $\mathrm{NH}_{3}+\mathrm{H} \rightleftharpoons \mathrm{NH} \mathrm{H}_{2}+\mathrm{H}_{2}$ & $6.8 \times 10^{3}$ & -0.8179 & -3068 \\
$\mathrm{~N} 24$ & $\mathrm{NH}_{2}+\mathrm{H} \rightleftharpoons \mathrm{NH}+\mathrm{H}_{2}$ & $3.4 \times 10^{1}$ & -0.3772 & 5764 \\
$\mathrm{~N} 25$ & $\mathrm{NH}_{2}+\mathrm{OH} \rightleftharpoons \mathrm{NH}+\mathrm{H}_{2} \mathrm{O}$ & $4.8 \times 10^{-1}$ & $-4.71 \times 10^{-2}$ & 13820 \\
$\mathrm{~N} 29$ & $\mathrm{NH}+\mathrm{H} \rightleftharpoons \mathrm{N}+\mathrm{H}_{2}$ & $8.1 \times 10^{-1}$ & -0.1602 & 12120 \\
$\mathrm{~N} 32$ & $\mathrm{NH}+\mathrm{OH} \rightleftharpoons \mathrm{N}+\mathrm{H}_{2} \mathrm{O}$ & $1.2 \times 10^{-2}$ & 0.1699 & 20170 \\
$\mathrm{~N} 34$ & $\mathrm{~N}+\mathrm{OH} \rightleftharpoons \mathrm{NO}+\mathrm{H}$ & $2.8 \times 10^{-2}$ & 0.3095 & 24580 \\
$\mathrm{~N} 35$ & $\mathrm{~N}+\mathrm{O} 2 \rightleftharpoons \mathrm{NO}+\mathrm{O}$ & $1.1 \times 10^{1}$ & $-9.64 \times 10^{-2}$ & 15930 \\
$\mathrm{~N} 36$ & $\mathrm{~N}+\mathrm{NO} \rightleftharpoons \mathrm{N}_{2}+\mathrm{O}$ & $5.0 \times 10^{-1}$ & $-9.70 \times 10^{-2}$ & 37710
\end{tabular}

${ }^{a}$ Numbering is the same as in Table 5 .

steps from Table 5 .

$$
\begin{gathered}
r_{\mathrm{HCN}}=w_{1}-w_{2}-w_{3}+w_{4}-w_{5}+w_{37}+w_{40}+w_{41}+w_{44} \\
+w_{46}+w_{47} \\
r_{\mathrm{NH}_{3}}=-w_{21}-w_{22}-w_{23} \\
r_{\mathrm{NO}}=w_{15}-w_{16}+w_{18}-w_{26}+w_{27}+w_{28}+w_{30}+w_{31}-w_{33} \\
+w_{34}+w_{35}-w_{36}-w_{39}-w_{40}-w_{41}-w_{42}-w_{43}-w_{45} \\
r_{\mathrm{N}_{2}}=w_{16}+w_{26}+w_{33}+w_{36}-w_{37}-w_{38}
\end{gathered}
$$

The net rate of reaction, $w_{i}$, is for the reaction $\mathrm{A}+\mathrm{B} \rightleftharpoons \mathrm{C}+\mathrm{D}$ calculated as

$$
w_{i}=k_{i}\left([\mathrm{~A}][\mathrm{B}]-[\mathrm{C}][\mathrm{D}] / K_{i}\right)
$$

Here, $k_{i}$ and $K_{i}$ are the forward rate constant and the equilibrium constant, respectively, for the $i$ th reaction. The equilibrium constants are listed in Table 6 . To solve the equations for the key nitrogen species, a number of steady-state equations must be solved for nitrogen radicals and intermediates. For HOCN and $\mathrm{CN}$, we obtain

$$
[\mathrm{HOCN}]=\frac{k_{\mathrm{f}, \mathrm{N} 5}[\mathrm{HCN}][\mathrm{OH}]}{k_{\mathrm{f}, \mathrm{N} 5} / K_{\mathrm{N} 5}[\mathrm{H}]+k_{\mathrm{f}, \mathrm{N} 6}[\mathrm{H}]+k_{\mathrm{f}, \mathrm{N} 7}[\mathrm{O}]+k_{\mathrm{f}, \mathrm{N} 8}[\mathrm{OH}]}
$$

$$
\begin{gathered}
{[\mathrm{CN}]=\left(k_{\mathrm{f}, \mathrm{N} 1} / K_{\mathrm{N} 1}[\mathrm{HCN}][\mathrm{H}]+k_{\mathrm{f}, \mathrm{N} 4} / K_{\mathrm{N} 4}[\mathrm{HCN}][\mathrm{OH}]\right.} \\
\left.+k_{\mathrm{f}, \mathrm{N} 38}[\mathrm{C}]\left[\mathrm{N}_{2}\right]+k_{\mathrm{f}, \mathrm{N} 42}[\mathrm{C}][\mathrm{NO}]\right) /\left(k_{\mathrm{f}, \mathrm{N} 1}\left[\mathrm{H}_{2}\right]+k_{\mathrm{f}, \mathrm{N} 4}\left[\mathrm{H}_{2} \mathrm{O}\right]\right. \\
\left.+k_{\mathrm{f}, \mathrm{N} 9}[\mathrm{OH}]+k_{\mathrm{f}, \mathrm{N} 10}\left[\mathrm{O}_{2}\right]\right)
\end{gathered}
$$

The equations for NCO and HNCO are solved simultaneously. The steady-state concentration for NCO is

$D_{\mathrm{NCO}}=k_{\mathrm{f}, \mathrm{N} 11} / K_{\mathrm{N} 11}\left[\mathrm{H}_{2} \mathrm{O}\right]+k_{\mathrm{f}, \mathrm{N} 12}\left[\mathrm{H}_{2}\right]+k_{\mathrm{f}, \mathrm{N} 13}[\mathrm{M}]+k_{\mathrm{f}, \mathrm{N} 14}[\mathrm{H}]$

$+k_{\mathrm{f}, \mathrm{N} 15}[\mathrm{O}]+k_{\mathrm{f}, \mathrm{N} 16}[\mathrm{NO}]$

$N_{1, \mathrm{NCO}}=\left([\mathrm{HOCN}]\left(k_{\mathrm{f}, \mathrm{N} 7}[\mathrm{O}]+k_{\mathrm{f}, \mathrm{N} 8}[\mathrm{OH}]\right)+[\mathrm{CN}]\left(k_{\mathrm{f}, \mathrm{N} 9}[\mathrm{OH}]\right.\right.$

$\left.\left.+k_{\mathrm{f}, \mathrm{N} 10}\left[\mathrm{O}_{2}\right]\right)+k_{\mathrm{f}, \mathrm{N} 2}[\mathrm{HCN}][\mathrm{O}]+k_{\mathrm{f}, \mathrm{N} 39}\left[\mathrm{CH}_{2}\right][\mathrm{NO}]\right) / D_{\mathrm{NCO}}$

$N_{2, \mathrm{NCO}}=\left(k_{\mathrm{f}, \mathrm{N} 11}[\mathrm{OH}]+k_{\mathrm{f}, \mathrm{N} 12} / K_{\mathrm{N} 12}[\mathrm{H}]\right) / D_{\mathrm{NCO}}$

$[\mathrm{NCO}]=N_{1, \mathrm{NCO}}+N_{2, \mathrm{NCO}}[\mathrm{HNCO}]$ 
The steady-state concentration of HNCO is

$$
\begin{aligned}
& D_{\mathrm{HNCO}}=k_{\mathrm{f}, \mathrm{N} 11}[\mathrm{OH}]+k_{\mathrm{f}, \mathrm{N} 12} / K_{\mathrm{N} 12}[\mathrm{H}]+k_{\mathrm{f}, \mathrm{N} 17}[\mathrm{M}] \\
& +k_{\mathrm{f}, \mathrm{N} 18}\left[\mathrm{O}_{2}\right]+k_{\mathrm{f}, \mathrm{N} 19}[\mathrm{H}]+k_{\mathrm{f}, \mathrm{N} 20}[\mathrm{O}] \\
& N_{1, \mathrm{HNCO}}=k_{\mathrm{f}, \mathrm{N} 6}[\mathrm{HOCN}][\mathrm{H}] / D_{\mathrm{HNCO}} \\
& N_{2, \mathrm{HNCO}}=\left(k_{\mathrm{f}, \mathrm{N} 11} / K_{\mathrm{N} 11}\left[\mathrm{H}_{2} \mathrm{O}\right]+k_{\mathrm{f}, \mathrm{N} 12}\left[\mathrm{H}_{2}\right]\right) / D_{\mathrm{HNCO}} \\
& {[\mathrm{HNCO}]=N_{1, \mathrm{HNCO}}+N_{2, \mathrm{HNCO}}[\mathrm{NCO}]}
\end{aligned}
$$

By solving eqs 19 and 20 simultaneously

$$
[\mathrm{NCO}]=\frac{N_{1, \mathrm{NCO}}+N_{2, \mathrm{NCO}} N_{1, \mathrm{HNCO}}}{1-N_{2, \mathrm{NCO}} N_{2, \mathrm{HNCO}}}
$$

The steady-state concentration of HCNO is found from

$$
[\mathrm{HCNO}]=\frac{k_{\mathrm{f}, \mathrm{N} 45}[\mathrm{HCCO}][\mathrm{NO}]}{k_{\mathrm{f}, \mathrm{N} 46}+k_{\mathrm{f}, \mathrm{N} 47}[\mathrm{H}]}
$$

The equations for the species $\mathrm{NH}_{2}, \mathrm{NH}$, and $\mathrm{N}$ are also solved simultaneously. The steady-state concentration for $\mathrm{NH}_{2}$ is

$$
\begin{aligned}
D_{\mathrm{NH}_{2}}= & k_{\mathrm{f}, \mathrm{N} 21} / K_{\mathrm{N} 21}[\mathrm{H}]+k_{\mathrm{f}, \mathrm{N} 22} / K_{\mathrm{N} 22}\left[\mathrm{H}_{2} \mathrm{O}\right]+k_{\mathrm{f}, \mathrm{N} 23} / K_{\mathrm{N} 23}\left[\mathrm{H}_{2}\right] \\
+ & k_{\mathrm{f}, \mathrm{N} 24}[\mathrm{H}]+k_{\mathrm{f}, \mathrm{N} 25}[\mathrm{OH}]+k_{\mathrm{f}, \mathrm{N} 26}[\mathrm{NO}] \\
N_{1, \mathrm{NH}_{2}}= & \left(k_{\mathrm{f}, \mathrm{N} 19}[\mathrm{HNCO}][\mathrm{H}]+\left[\mathrm{NH}_{3}\right]\left(k_{\mathrm{f}, \mathrm{N} 21}[\mathrm{M}]+k_{\mathrm{f}, \mathrm{N} 22}[\mathrm{OH}]\right.\right. \\
& \left.+k_{\mathrm{f}, \mathrm{N} 23}[\mathrm{H}]\right) / D_{\mathrm{NH}_{2}} \\
N_{2, \mathrm{NH}_{2}}= & \left(k_{\mathrm{f}, \mathrm{N} 24} / K_{\mathrm{N} 24}\left[\mathrm{H}_{2}\right]+k_{\mathrm{f}, \mathrm{N} 25} / K_{\mathrm{N}_{25}}\left[\mathrm{H}_{2} \mathrm{O}\right]\right) / D_{\mathrm{NH}_{2}} \\
{\left[\mathrm{NH}_{2}\right]=} & N_{1, \mathrm{NH}_{2}}+N_{2, \mathrm{NH}_{2}}[\mathrm{NH}]
\end{aligned}
$$

The steady-state concentration for $\mathrm{NH}$ is

$$
\begin{gathered}
D_{\mathrm{NH}}=k_{\mathrm{f}, \mathrm{N} 24} / K_{\mathrm{N} 24}\left[\mathrm{H}_{2}\right]+k_{\mathrm{f}, \mathrm{N} 25} / K_{\mathrm{N} 25}\left[\mathrm{H}_{2} \mathrm{O}\right] \\
+\left(k_{\mathrm{f}, \mathrm{N} 27}+k_{\mathrm{f}, \mathrm{N} 28}\right)\left[\mathrm{O}_{2}\right]+k_{\mathrm{f}, \mathrm{N} 29}[\mathrm{H}]+k_{\mathrm{f}, \mathrm{N} 30}[\mathrm{O}] \\
+\left(k_{\mathrm{f}, \mathrm{N} 31}+k_{\mathrm{f}, \mathrm{N} 32}\right)[\mathrm{OH}]+k_{\mathrm{f}, \mathrm{N} 33}[\mathrm{NO}] \\
N_{1, \mathrm{NH}}=\left(k_{\mathrm{f}, \mathrm{N} 3}[\mathrm{HCN}][\mathrm{O}]+k_{\mathrm{f}, \mathrm{N} 14}[\mathrm{NCO}][\mathrm{H}]\right. \\
\left.+[\mathrm{HNCO}]\left(k_{\mathrm{f}, \mathrm{N} 17}[\mathrm{M}]+k_{\mathrm{f}, \mathrm{N} 20}[\mathrm{O}]\right)\right) / D_{\mathrm{NH}} \\
N_{2, \mathrm{NH}}=\left(k_{\mathrm{f}, \mathrm{N} 29} / K_{\mathrm{N} 29}\left[\mathrm{H}_{2}\right]+k_{\mathrm{f}, \mathrm{N} 32} / K_{\mathrm{N} 32}\left[\mathrm{H}_{2} \mathrm{O}\right]\right) / D_{\mathrm{NH}} \\
N_{3, \mathrm{NH}}=\left(k_{\mathrm{f}, \mathrm{N} 24}[\mathrm{H}]+k_{\mathrm{f}, \mathrm{N} 25}[\mathrm{OH}] / D_{\mathrm{NH}}\right. \\
{[\mathrm{NH}]=N_{1, \mathrm{NH}}+N_{2, \mathrm{NH}}[\mathrm{N}]+N_{3, \mathrm{NH}}\left[\mathrm{NH}_{2}\right]}
\end{gathered}
$$

Finally, the steady-state concentration of $\mathrm{N}$ is

$$
\begin{aligned}
& D_{\mathrm{N}}=k_{\mathrm{f}, \mathrm{N} 29} / K_{\mathrm{N} 29}\left[\mathrm{H}_{2}\right]+k_{\mathrm{f}, \mathrm{N} 32} / K_{\mathrm{N} 32}\left[\mathrm{H}_{2} \mathrm{O}\right]+k_{\mathrm{f}, \mathrm{N} 34}[\mathrm{OH}] \\
& +k_{\mathrm{f}, \mathrm{N} 35}\left[\mathrm{O}_{2}\right]+k_{\mathrm{f}, \mathrm{N} 36}[\mathrm{NO}]+k_{\mathrm{f}, \mathrm{N} 44}\left[\mathrm{CH}_{3}\right] \\
& \quad N_{1, \mathrm{~N}}=\left(k_{\mathrm{f}, \mathrm{N} 13}[\mathrm{NCO}][\mathrm{M}]+[\mathrm{NO}]\left(k_{\mathrm{f}, \mathrm{N} 34} / K_{\mathrm{N} 34}[\mathrm{H}]\right.\right. \\
& \left.+k_{\mathrm{f}, \mathrm{N} 35} / K_{\mathrm{N} 35}[\mathrm{O}]\right)+k_{\mathrm{f}, \mathrm{N} 36} / K_{\mathrm{N} 36}\left[\mathrm{~N}_{2}\right][\mathrm{O}]+k_{\mathrm{f}, \mathrm{N} 37}[\mathrm{CH}]\left[\mathrm{N}_{2}\right] \\
& \left.+k_{\mathrm{f}, \mathrm{N} 38}[\mathrm{C}]\left[\mathrm{N}_{2}\right]+k_{\mathrm{f}, \mathrm{N} 43}[\mathrm{C}][\mathrm{NO}]\right) / D_{\mathrm{N}} \\
& N_{2, \mathrm{~N}}=\left(k_{\mathrm{f}, \mathrm{N} 29}[\mathrm{H}]+k_{\mathrm{f}, \mathrm{N} 32}[\mathrm{OH}]\right) / D_{\mathrm{N}} \\
& {[\mathrm{N}]=N_{1, \mathrm{~N}}+N_{2, \mathrm{~N}}[\mathrm{NH}]}
\end{aligned}
$$

Equations 23-25 are solved simultaneously.

$$
[\mathrm{NH}]=\frac{N_{1, \mathrm{NH}}+N_{2, \mathrm{NH}} N_{1, \mathrm{~N}}+N_{3, \mathrm{NH}} N_{1, \mathrm{NH}_{2}}}{1-N_{2, \mathrm{NH}} N_{2, \mathrm{~N}}-N_{3, \mathrm{NH}} N_{2, \mathrm{NH}_{2}}}
$$
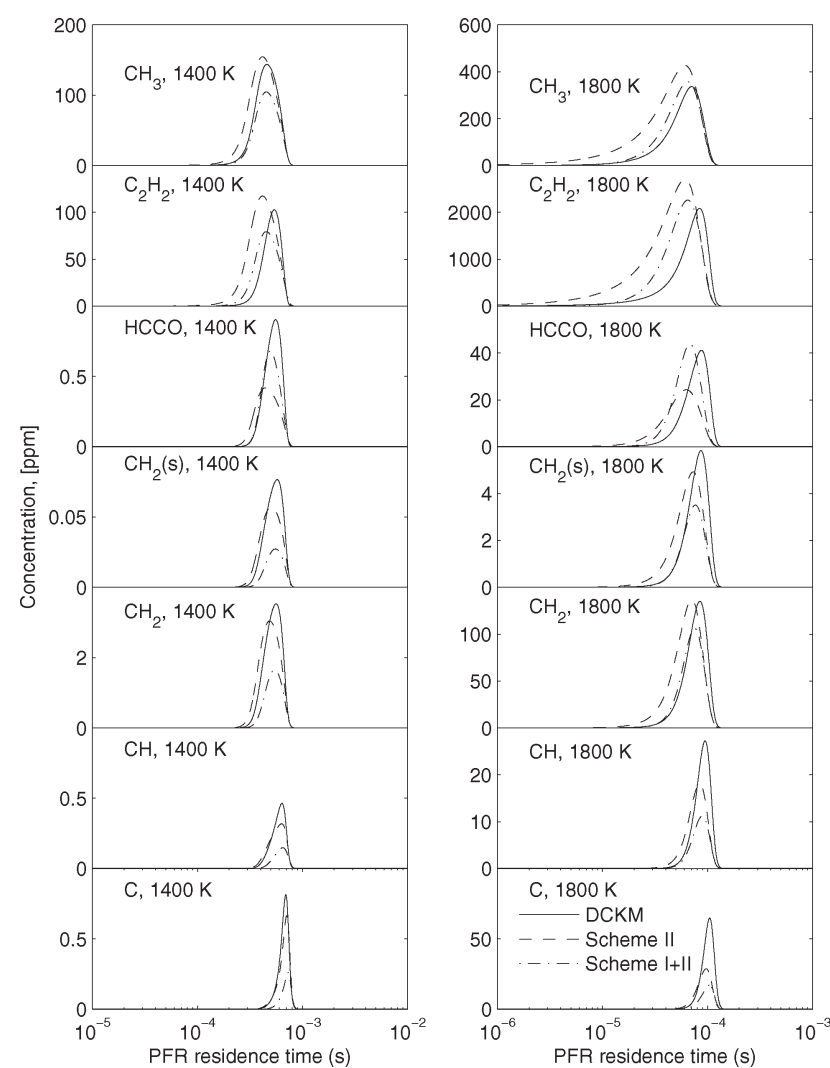

Figure 2. Radical concentrations during combustion of bituminous coal at two temperatures and reburning conditions. $\lambda=0.8$, and $\mathrm{O}_{2}$ inlet concentration $=1 \mathrm{vol} \%$.

\section{Results and Discussion}

In the following, we define the schemes used in modeling as follows: DCKM, detailed chemical kinetics model; ${ }^{20,23} \mathrm{I}$, prediction of $\mathrm{H}, \mathrm{O}$, and $\mathrm{OH}$ from the semi-empirical correlations of ref 27; II, prediction of hydrocarbon radicals from eqs $1-11$; and III, prediction of the nitrogen chemistry from eqs $12-26$

Figures 2 and 3 show predictions of hydrocarbon radical concentrations during combustion of bituminous coal and biomass, respectively. The figures compare modeling predictions from three levels of calculations: with DCKM, with scheme II and DCKM (the $\mathrm{O} / \mathrm{H}$ radical pool, as well as $\mathrm{CH}_{4}$, $\mathrm{O}_{2}, \mathrm{CO}_{2}, \mathrm{H}_{2} \mathrm{O}, \mathrm{H}_{2}$, and $\mathrm{N}_{2}$, from the detailed mechanism), and with schemes I, II, and DCKM $\left(\mathrm{CH}_{4}, \mathrm{O}_{2}, \mathrm{CO}_{2}, \mathrm{H}_{2} \mathrm{O}, \mathrm{H}_{2}\right.$, and $\mathrm{N}_{2}$ from the detailed model). The results show that the peak hydrocarbon radical concentrations are mostly predicted within a factor of 2 compared to the reference calculations. In general, the accuracy is better at higher temperatures and not too fuel-rich conditions $(\lambda \geq 0.6)$. Also, radicals, such as $\mathrm{CH}_{3}$ and $\mathrm{HCCO}$, formed directly from stable species are predicted more accurately than radicals, such as $\mathrm{CH}$ and $\mathrm{C}$, estimated from a sequence of steady-state approximations. Bituminous coal and biomass represent extremes in terms of volatile composition and rank (Table 1). Still, the deviations from the reference calculations are similar for the two fuels.

The results indicate that the approximations in the simplified hydrocarbon radical scheme are satisfactory. However, when this scheme is combined with the semi-empirical correlations for the $\mathrm{O} / \mathrm{H}$ radicals, the modeling predictions become less accurate. 

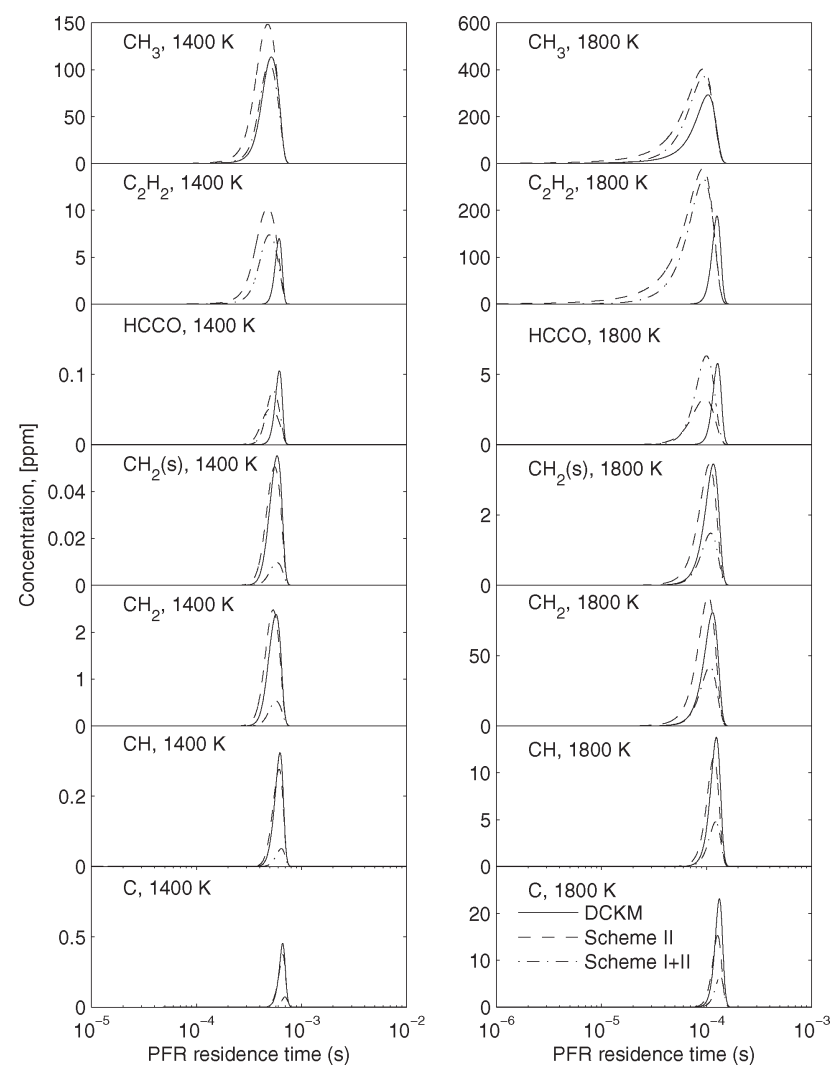

Figure 3. Radical concentrations during combustion of biomass at two temperatures and reburning conditions. $\lambda=0.8$, and $\mathrm{O}_{2}$ inlet concentration $=1 \mathrm{vol} \%$

The empirical equations developed to describe the formation and consumption of the hydrocarbon (scheme II) and $\mathrm{O} /$ $\mathrm{H}$ radical pool (scheme I) are combined with the reduced scheme for reactive nitrogen conversion established in this work (scheme III) to yield a model for volatile nitrogen reburning in solid fuel combustion. The following figures compare modeling predictions from three levels of calculations: with DCKM, with scheme III and DCKM (all radicals as well as $\mathrm{CH}_{4}, \mathrm{O}_{2}, \mathrm{CO}_{2}, \mathrm{H}_{2} \mathrm{O}$, and $\mathrm{H}_{2}$ from the detailed mechanism), and with schemes I-III and DCKM $\left(\mathrm{CH}_{4}, \mathrm{O}_{2}\right.$, $\mathrm{CO}_{2}, \mathrm{H}_{2} \mathrm{O}$, and $\mathrm{H}_{2}$ from the detailed model).

Figures 4 and 5 show results for reburning with volatiles from biomass and bituminous coal, respectively, as a function of stoichiometry $(0.6 \leq \lambda \leq 1.0)$ and temperature $(1400-1800 \mathrm{~K})$. Under the conditions investigated, the reduction in $\mathrm{NO}$ is modest. It increases as the stoichiometry becomes more fuel-rich, while the temperature has only a small impact. The combined simplified model (I + II + III) provides a satisfactory estimate of the NO concentration for both fuels.

Figure 6 compares predictions with the simplied model to reference calculations with the full mechanism for all four fuels at the two temperatures and $\lambda=0.6$. Again, there is good agreement between the full mechanism and the simplified schemes. The calculations indicate that the major hydrocarbon radicals, $\mathrm{CH}_{3}$ and $\mathrm{HCCO}$, are responsible for most of the reduction in NO. For this reason, the shortcomings in predicting the concentrations of the smaller hydrocarbon radicals (Figures 2 and 3) do not significantly deteriorate modeling predictions.

3.1. Practical Application. It is most efficient in terms of computational efforts to conduct the calculations on the
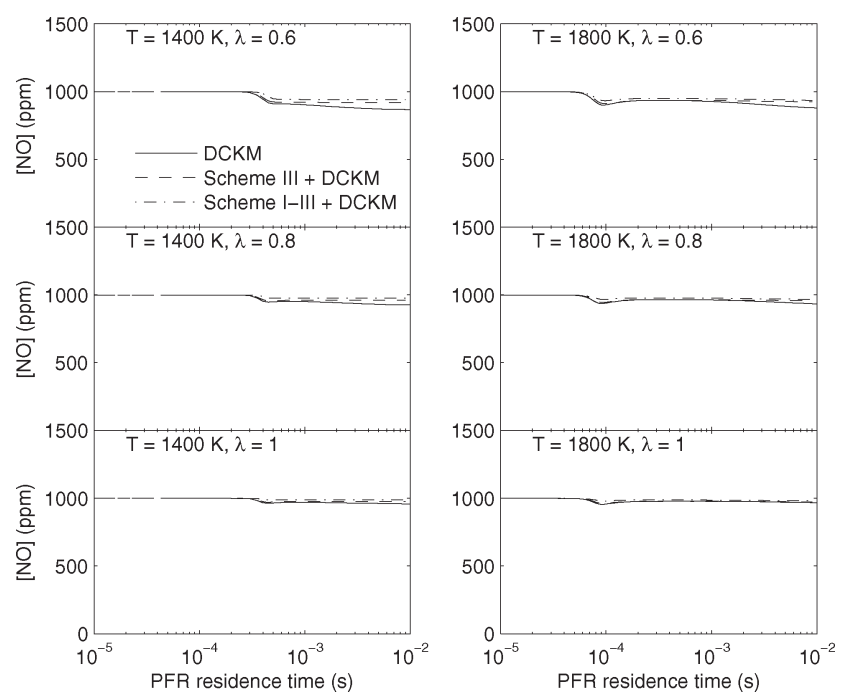

Figure 4. Predictions of $\mathrm{NO}$ reburning with biomass volatiles in $\mathrm{N}_{2} / \mathrm{O}_{2}$ mixtures with $1000 \mathrm{ppm} \mathrm{NO}$ and $1 \mathrm{vol} \% \mathrm{O}_{2}$ in the inlet. The calculations are conducted under isothermal conditions. The figure compares modeling predictions from three levels of calculations: with DCKM, with scheme III and DCKM, and with schemes I-III and DCKM.
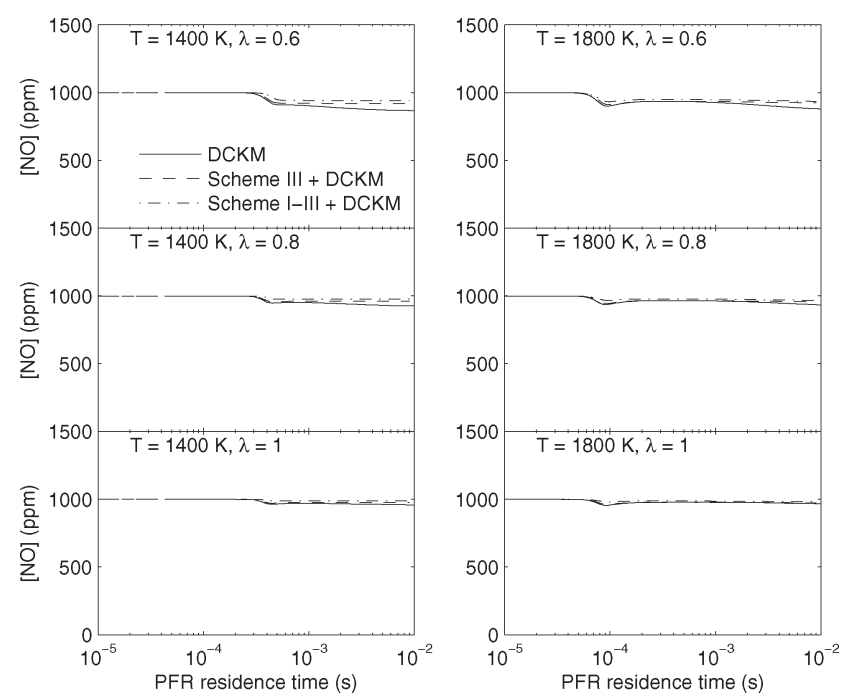

Figure 5. Predictions of NO reburning with bituminous coal volatiles in $\mathrm{N}_{2} / \mathrm{O}_{2}$ mixtures with $1000 \mathrm{ppm} \mathrm{NO}$ and $1 \mathrm{vol} \% \mathrm{O}_{2}$ in the inlet. The calculations are conducted under isothermal conditions. The figure compares modeling predictions from three levels of calculations: with DCKM, with scheme III and DCKM, and with schemes I-III and DCKM.

nitrogen chemistry as either post-processing or activated in a final iteration upon convergence of the main scalars., ${ }^{27}$ These approaches imply that the nitrogen chemistry does not affect the overall flow pattern and temperature. Even though trace species including NO have been reported to affect emissions and combustion rates ${ }^{36}$ the assumption is justifiable because only a small fraction of the overall gas flow is involved in the active nitrogen chemistry.

To facilitate the practical implementation of the $\mathrm{NO}_{x}$ model, i.e., schemes I (the $\mathrm{O} / \mathrm{H}$ radical mode ${ }^{27}$ ), II (the hydrocarbon

(36) Glarborg, P. Proc. Combust. Inst. 2007, 31, 77-98. 

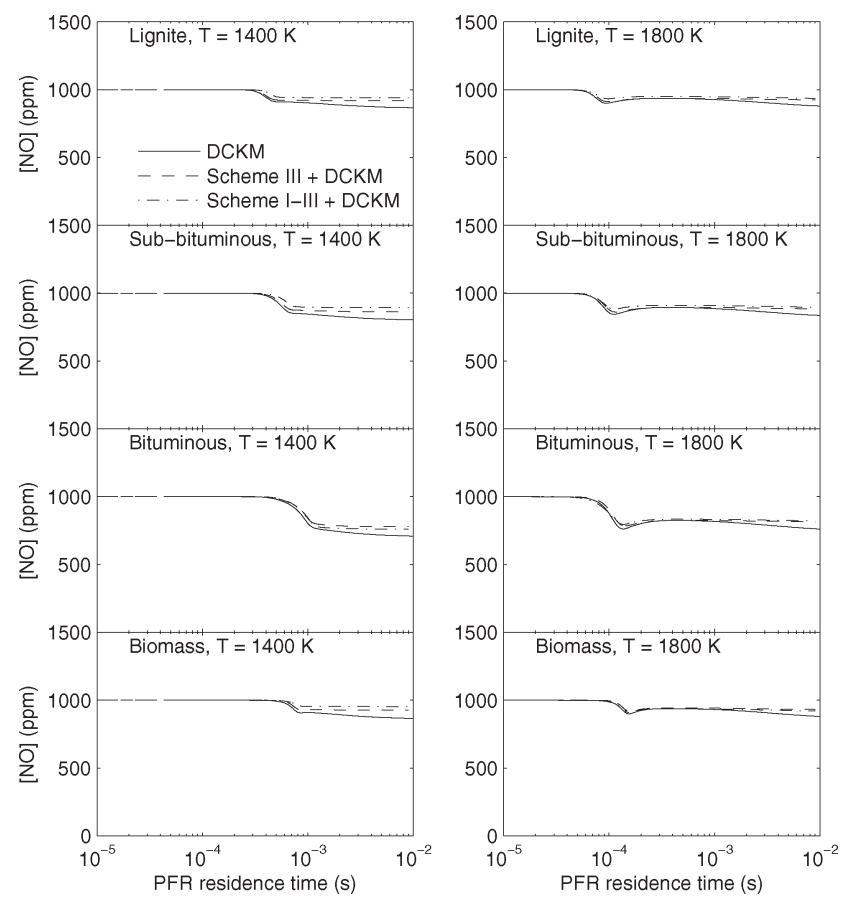

Figure 6. Predictions of $\mathrm{NO}$ reburning in the $\mathrm{N}_{2} / \mathrm{O}_{2}$ mixture with 1000 ppm NO and $1 \mathrm{vol} \% \mathrm{O}_{2}$ in the inlet and $\lambda=0.6$. The calculations are conducted under isothermal conditions. The figure compares modeling predictions from three levels of calculations: with DCKM, with scheme III and DCKM, and with schemes I-III and DCKM.

radical model), and III (the $\mathrm{N}$ scheme), we discuss below the implications for the main calculation and the post-processing. Matlab scripts for the $\mathrm{NO}_{x}$ model are included as Supporting Information.

3.1.1. Main Combustion Calculation. The main calculation establishes the flow field, the temperature field, and the major species concentrations. The main issue for the present purpose is the choice of models for pyrolysis and volatile oxidation. Dependent upon the complexity of the volatile oxidation model, the major species may comprise just a single volatile component, together with final products $\mathrm{CO}_{2}$ and $\mathrm{H}_{2} \mathrm{O}$, or a more complete set of fuel components, intermediates, and products. The use of a simple one- or twostep volatile oxidation model is compatible with the $\mathrm{NO}_{x}$ model, as described below. However, while this approach will save computational resources, it could also be expected to yield less accurate modeling predictions compared to using a more advanced pyrolysis/volatile oxidation model. If a more complex model is chosen for pyrolysis and volatile oxidation, it is important that it is compatible with the $\mathrm{NO}_{x}$ model in terms of composition of the volatiles. This issue is discussed in detail below.

3.1.2. Nitrogen Chemistry Calculation. The calculations of the nitrogen chemistry can be conducted in a post-processing step. The main calculation provides information on flow field, temperature field, and major species concentrations. However, the time-dependent concentration profiles of $\mathrm{O}_{2}$, $\mathrm{H}_{2}$, and $\mathrm{H}_{2} \mathrm{O}$ for each cell, required as input for schemes I-III, are not available from the main calculation. For this reason, the post-processing calculations must combine a volatile oxidation model with the radical schemes (I and II) and the $\mathrm{N}$ oxidation model (III).

The fitting parameters in the functional form chosen for the radical schemes are fuel-specific, in both I and II. The volatile composition entering the post-processing calculation should be consistent with the data for one of the fuels listed in Table 1.27 The data in the table are categorized according to fuel rank (bituminous coal, sub-bituminous coal, lignite, and biomass) and cover most solid fuels of importance. The correlations for the four fuels developed in this work may be applicable to other fuels within the same rank, because minor variations in volatile composition will only have a small impact on modeling predictions. If the pyrolysis model used in the main calculation provides only a simplified volatile composition, the split between the chosen fuel components (hydrocarbons, $\mathrm{H}_{2}$, and $\mathrm{CO}$ ) needed for the post-processing computations must be estimated algebraically.

The volatile model must describe the oxidation of the fuel elements, i.e., hydrocarbons, hydrogen, and carbon monoxide. The estimated concentrations of $\mathrm{O}_{2}, \mathrm{H}_{2}$, and $\mathrm{H}_{2} \mathrm{O}$ for each time step are then used as input to schemes I-III. The choice of the volatile oxidation model will affect the computational effort as well as the expected accuracy. ${ }^{27,37}$ The model could be a collection of global reactions, i.e., a single oxidation reaction for each fuel component in the volatiles. Rate constants for global oxidation steps are available for a range of fuel components ${ }^{24,38,39}$ and may be readily available in the chosen CFD software. Even in a combination with a set of global reactions for volatile oxidation, the present model (schemes I-III) offers an improved prediction of the nitrogen chemistry compared to CFD standard models. For a more accurate prediction, the Jones and Lindstedt global four-step combustion mechanism ${ }^{25}$ can be implemented. Four-step mechanisms are offered for several hydrocarbon fuels and also involve steps for oxidation of $\mathrm{H}_{2}$ and $\mathrm{CO}$.

\section{Conclusion}

In the present work, an analytically reduced nitrogen scheme was combined with simplified correlations for estimation of $\mathrm{O} / \mathrm{H}$ and hydrocarbon radicals. This way, the predictive capability of the analytically reduced models is combined with the robustness and speed of more simplified schemes. Correlations were derived for volatile compositions representative of solid fuels ranging from bituminous coal to biomass, for temperatures of 1200-2000 K and excess air ratios in the range of $0.6 \leq \lambda \leq 2.0$. The combined model is tested against reference calculations with a comprehensive mechanism. The results indicate that the approximations in the simplified hydrocarbon radical scheme are satisfactory. However, when this scheme is combined with the semiempirical correlations for the $\mathrm{O} / \mathrm{H}$ radicals, the modeling predictions for the radicals become less accurate. Despite these deviations, the combined model provides a satisfactory prediction of NO under reburning conditions over the range of fuels, temperatures, and stoichiometries tested.

Acknowledgment. The work was funded by Vattenfall Research and Development AB and DONG Energy A/S.

Supporting Information Available: Matlab scripts for the $\mathrm{NO}_{x}$ model. This material is available free of charge via the Internet at http://pubs.acs.org.

(37) Andersen, J.; Rasmussen, C. L.; Giselsson, T.; Glarborg, P. Energy Fuels 2009, 23, 1379-1389.

(38) Hautman, D. J.; Dryer, F. L.; Schug, K. P.; Glassman, I. Combust. Sci. Technol. 1981, 25, 219-235.

(39) Babushok, V. I.; Dakdancha, A. N. Combust., Explos. Shock Waves 1993, 29, 464-489. 